\title{
Structural and Intensity Changes of Concentric Eyewall Typhoons in the Western North Pacific Basin
}

\author{
Yi-TiNG YANG AND HunG-CHI KUO \\ Department of Atmospheric Sciences, National Taiwan University, Taipei, Taiwan \\ ERIC A. Hendricks AND MELinda S. PENG \\ Naval Research Laboratory, Monterey, California
}

(Manuscript received 31 August 2012, in final form 7 February 2013)

\begin{abstract}
An objective method is developed to identify concentric eyewalls (CEs) for typhoons using passive microwave satellite imagery from 1997 to 2011 in the western North Pacific basin. Three CE types are identified: a CE with an eyewall replacement cycle (ERC; 37 cases), a CE with no replacement cycle (NRC; 17 cases), and a CE that is maintained for an extended period (CEM; 16 cases). The inner eyewall (outer eyewall) of the ERC (NRC) type dissipates within $20 \mathrm{~h}$ after CE formation. The CEM type has its CE structure maintained for more than $20 \mathrm{~h}$ (mean duration time is $31 \mathrm{~h}$ ). Structural and intensity changes of CE typhoons are demonstrated using a $T$-Vmax diagram (where $T$ is the brightness temperature and Vmax is the best-track estimated intensity) for a time sequence of the intensity and convective activity (CA) relationship. While the intensity of typhoons in the ERC and CEM cases weakens after CE formation, the CA is maintained or increases. In contrast, the CA weakens in the NRC cases. The NRC (CEM) cases typically have fast (slow) northward translational speeds and encounter large (small) vertical shear and low (high) sea surface temperatures. The CEM cases have a relatively high intensity $\left(63 \mathrm{~m} \mathrm{~s}^{-1}\right)$, and the moat size $(61 \mathrm{~km})$ and outer eyewall width $(70 \mathrm{~km})$ are approximately $50 \%$ larger than the other two categories. Both the internal dynamics and environmental conditions are important in the CEM cases, while the NRC cases are heavily influenced by the environment. The ERC cases may be dominated by the internal dynamics because of more uniform environmental conditions.
\end{abstract}

\section{Introduction}

One of the great challenges associated with tropical cyclone (TC) prediction is the large variability in structure and intensity changes. One dynamical mechanism known to produce such variability is the concentric eyewall (CE) formation and the eyewall replacement cycle (ERC) in TCs (Willoughby et al. 1982; Black and Willoughby 1992; Kuo et al. 2009; Sitkowski et al. 2011). Strong TCs are often observed to have a CE structure that has an inner eyewall and an outer eyewall separated by a convective minimum region, the moat (Willoughby et al. 1982). The ERC is defined as the time period from the formation of the secondary eyewall, to its contraction,

Corresponding author address: Hung-Chi Kuo, Department of Atmospheric Sciences, National Taiwan University, No. 1, Sec. 4, Roosevelt Road, Taipei 10617, Taiwan.

E-mail: kuo@as.ntu.edu.tw and finally to the replacement of the original decaying inner eyewall. Willoughby et al. (1982) investigated the ERC dynamics. Their results suggested that a local tangential wind maximum is associated with the outer eyewall and the most rapid increase in wind speed lies on the inward side of the wind maximum. The outer wind maximum thus contracts and intensifies and the inner eyewall weakens and eventually vanishes. Previous observational studies also indicate that the secondary eyewall can act as a barrier to the moisture inflow to the inner eyewall (e.g., Barnes et al. 1983; Barnes and Powell 1995; Samsury and Zipser 1995). The ERC time scale in numerical models such as Terwey and Montgomery (2008), Qiu et al. (2010), and Zhou and Wang (2009) are in the range of $6-18 \mathrm{~h}$. The radar observations of Typhoon Lekima (Kuo et al. 2004) indicated that the ERC time is approximately $6 \mathrm{~h}$. Many theories allude to the influences of both synoptic-scale environmental conditions and mesoscale processes in CE formation. Both 
the internal and external dynamics may play important roles in CE formation. Nong and Emanuel (2003) showed that the CE may form because of favorable ambient environmental conditions or external forcing and a wind-induced surface heat exchange (WISHE) instability. Examples of internal dynamics include propagating vortex Rossby waves that interact with a critical radius (Montgomery and Kallenbach 1997; Peng et al. 2009) and axisymmetrization during a binary vortex interaction (Kuo et al. 2004, 2008). The binary vortex interaction dynamics highlight the axisymmetrization of finite-area positive vorticity perturbations around a strong and tight core to produce the CE structure. Kuo et al. (2008) further suggested that vorticity skirts outside the TC core may produce various sizes of the $\mathrm{CE}$ through the mechanism of binary vortex interactions. This may explain satellite microwave observations showing a wide range of radii for CE. Terwey and Montgomery (2008) proposed the existence of a region with moderate horizontal strain deformation and a sufficient low-level radial potential vorticity gradient associated with the primary swirling flow, moist convective potential, and a wind-moisture feedback process at the air-sea interface to form the secondary eyewall. Recently, Huang et al. (2012) suggested that the broadening of the radial tangential wind profile above the boundary layer in a symmetric fashion can lead to boundary layer convergence and inflow. The progressive strengthening of the boundary layer inflow, and the unbalanced boundary layer response, may lead to secondary eyewall formation.

Sitkowski et al. (2011) used flight-level data to study the ERC process in the Atlantic basin. They suggested that the ERC time requirement, the intensity change, and the radii change have large variances. The ERC of individual storms can deviate greatly from the mean characteristics. The key features of CE are not only associated with intensity but also structural changes. Maclay et al. (2008) used the low-level area-integrated kinetic energy to show that while the intensity weakens during the ERC, the integrated kinetic energy and the TC size increases. Their results suggest that secondary eyewall formation and ERC are dominated by internal dynamical processes. The intensity and structural changes can best be demonstrated by the $K-\mathrm{Vmax}$ diagram (Maclay et al. 2008); a diagram in which the ordinate is the integrated kinetic energy deviation inside a radius of $200 \mathrm{~km}$ and the abscissa is the maximum tangential wind. The deviation is calculated from an areal kinetic energy regression with intensity for TC in Atlantic basin. Thus, the $K-V m a x$ diagrams give not only the areal kinetic energy change in the secondary eyewall formation but also the kinetic energy difference as compared to the typical storms. ${ }^{1}$ The areal kinetic energy is typically calculated from the aircraft reconnaissance data. Both Maclay et al. (2008) and Sitkowski et al. (2011) indicated that the ERC is a primary mechanism for storm growth, with both the integrated kinetic energy and the TC size increasing during the process.

The availability of passive microwave data in the last decade has made it possible to reveal more clearly the CE structure in TCs, which had not been previously possible with visible and infrared satellite data. Using passive microwave data between 1997 and 2002, Hawkins and Helveston (2004) suggested that CEs exist with a much higher percentage (i.e., $80 \%$ ) in intense TCs [maximum wind $>120 \mathrm{kt}\left(\sim 62 \mathrm{~m} \mathrm{~s}^{-1}\right)$ ] than previously realized in the western North Pacific basin. As further noted by Hawkins et al. (2006), there were many more CE cases with large radius in the western Pacific than in other basins. Kuo et al. (2009) studied the intensity change and moat dynamics of CE typhoons using passive microwave data and best-track data in the western North Pacific basin between 1997 and 2006. Their results indicate that only half $(51 \%)$ of $\mathrm{CE}$ formation cases follow the intensity change pattern of the ERC, where the intensity increases $24 \mathrm{~h}$ before CE formation and then weakens in the next $24 \mathrm{~h}$. The rest of the cases do not follow the ERC - they often possess a larger moat size and their inner eyewalls are still identifiable for $24 \mathrm{~h}$ or longer.

Hawkins and Helveston (2008) gave examples of different modes of CE structure, including the ERC (Black and Willoughby 1992), triple eyewalls (McNoldy 2004), ERCs that are repeated multiple times, ERCs that are interrupted by vertical shear and landfall, and cases where an outer eyewall forms at a large radius and remains in a CE structure for a long period of time. The different CE modes appear to have profound impacts on intensity and structural forecasts and warrant more quantitative analysis and documentation. The purpose of the present study is to quantitatively examine these structural and intensity changes of CE typhoons in the western North Pacific basin in an objective manner. Microwave satellite imagery, best-track data from the Joint Typhoon Warning Center (JTWC), and data from the Statistical Typhoon Intensity Prediction Scheme (STIPS; Knaff et al. 2005) are used between 1997 and 2011 for this study. The development of the STIPS model closely follows the development of the Statistical Hurricane Intensity Prediction Scheme (SHIPS)

\footnotetext{
${ }^{1}$ Musgrave et al. (2012) use a similar $K-\mathrm{Vmax}$ diagram in which the ordinate is the integrated kinetic energy inside a radius of $1000 \mathrm{~km}$ and the abscissa is the maximum tangential wind.
} 
model in the Atlantic and east Pacific tropical cyclone basins as described in DeMaria and Kaplan (1999) and DeMaria et al. (2005). An objective method is developed to identify typhoons with a $\mathrm{CE}$ and then categorize the CEs in a systematic manner. The data and methodology are presented in section 2 . The observed structural and intensity changes of the different CE classifications are presented in section 3. The environmental factors and the analysis of structural characteristics such as the moat width and outer eyewall width are presented in section 4. Finally, the conclusions are provided in section 5 .

\section{Data and methodology}

\section{a. Data source}

Microwave satellite data are used to examine the characteristics of typhoons with CEs in the western North Pacific basin between 1997 and 2011. The microwave satellite data used are the passive Special Sensor Microwave Imager (SSM/I) 85-GHz horizontal polarized orbital imagery and passive Tropical Rainfall Measuring Mission (TRMM) Microwave Imager (TMI) data from the polar-orbiting TRMM satellite (Kummerow et al. 1998). These data were obtained from the Naval Research Laboratory (NRL) Marine Meteorology Division in Monterey, California (Hawkins et al. 2001). The swath width of the TMI data $(759 \mathrm{~km})$ generally is smaller than the SSM/I data $(1394 \mathrm{~km})$ but the resolution $(5-37 \mathrm{~km})$ is higher than the SSM/I data $(12.5-50 \mathrm{~km}$; Kidder et al. 2000). In the microwave data, the $85.5-\mathrm{GHz}$ channel was used to identify CEs because it is a good indicator for ice above the freezing level in tropical deep convection. For example, Spencer et al. (1989) showed that the blackbody brightness temperature $T_{B}$ of the Advanced Microwave Moisture Sounder (AMMS) matches the rain intensity of an ER-2 reconnaissance flight. A good correlation between the $19.35-\mathrm{GHz} T_{B}$ warming (dominated by rain emission below the freezing level) and $85.5-\mathrm{GHz} T_{B}$ cooling (dominated by ice scattering above the freezing level) was obtained by the SSM/I in tropical oceanic precipitation systems.

In addition to SSM/I and TMI data, the Advanced Microwave Scanning Radiometer for Earth Observing System (AMSR-E) data are available starting from October 2003 and Special Sensor Microwave Imager/ Sounder (SSM/IS) data from August 2005. The data length is shorter than that of the SSM/I and TMI data. Moreover, neither have $85 \mathrm{GHz}$ and the brightness temperature changes may vary by $8-11 \mathrm{~K}$ when converted from 89 to $91 \mathrm{GHz}$. To give a 15 -yr comprehensive study for the CE climatology in a consistent manner, we currently concentrate on the SSM/I and TMI data.
The Dvorak technique is currently used by JTWC to estimate TC intensity. The JTWC also use the Geostationary Meteorological Satellite (GMS) infrared and visible cloud images to estimate intensity in the besttrack data. The maximum 1-min sustained wind speed was derived using the Dvorak $T$ number. The individual $T$ number represents a typical intensity change based on climatology. The Advanced Dvorak Technique (ADT), a fully automated and objective version of the original Dvorak technique, has been used by JTWC since 2004. On average, the $T$ number estimation is better than older version of Dvorak technique (Velden et al. 2006; Olander and Velden 2007).

We have compared the JTWC intensity with that of Japan Meteorological Agency (JMA) at a time interval of $24 \mathrm{~h}$ before and after the CE formation. The JMA intensity is generally weaker than that of JTWC by some $20 \%$ because JMA uses a 10-min sustained wind while JTWC uses a 1-min sustained wind. On the other hand, the correlation of the two intensities is high with $R^{2}=0.9$. The correlation degraded $12 \mathrm{~h}$ before the $\mathrm{CE}$ formation to $R^{2}=0.8$. The $R^{2}$ correlation further decreased to 0.7 after the CE formation. This may indicate the CE formation may affect the intensity determination in different agencies. Recent field experiments such as the Tropical Cyclone Structure 2008 (TCS-08) and the Impact of Typhoon on the Ocean in the Pacific 2010 (ITOP10) aircraft data (Hawkins and Velden 2011) may be used to improve the satellite intensity estimation.

The environmental parameters used in this study are the 850-700- and 700-500-hPa relative humidity (RH), vertical wind shear (850-200 hPa), sea surface temperature (SST), ocean heat content (OHC), and maximum potential intensity (MPI). These data are obtained from STIPS database.

\section{b. Image processing}

The microwave satellite images published on the NRL website are reprocessed using the Backus-Gilbert theory of Poe (1990) to create high-resolution (1-2 km) products that can assist in defining inner-storm structural details (Hawkins and Helveston 2004; Hawkins et al. 2006). The resampling method of Poe (1990) is optimal in the sense that it attempts to preserve the spatial resolution of the antenna gain function associated with the sampled radiometer data. These images are stored as $800 \times 800$ pixel color jpeg files that are composed with red (R), green (G), and blue (B) colors. Owing to the orbital satellite swath width, some portion of the image may not have data. The missing areas are filled with the nearest pass of geostationary satellite imageries (Hawkins et al. 2001). These parts of pixels have the same $R, G$, and $B$ values as the visible and 
infrared channels that are displayed in grayscale. Moreover, the coast lines and coordinate lines are shown by white color with R, G, and B color values of 255 . In processing the data, the pixels that contain geostationary satellite imagery, coast lines and coordinate lines are first removed from the NRL jpeg files. Subsequently, the remaining pixels are converted into $\mathrm{R}, \mathrm{G}$, and $\mathrm{B}$ components in order to determine the high resolution $T_{B}$ based on the color table provided in the satellite picture.

\section{c. Concentric eyewall recognition}

To identify CE typhoons, the $T_{B}$ dataset is transformed from Cartesian to polar coordinates with the TC center as the origin. The TC center is determined based on the JTWC best-track data at the time closest to satellite observation as the TC center. Occasionally, some subjective adjustment was required to fix the TC center when the time gap between the JTWC data and satellite data was large. In some cases, the eye centers were difficult to determine precisely either because of the irregular shape of the eye or in the situation of very large TC eye. We have performed tests to show that the radial profiles are not sensitive to a five pixel (or $10 \mathrm{~km}$ ) shift in the TC position. There were also difficulties in defining the TC center when the TC was weak. These weak TCs, however, almost always occurred at the beginning or near the end of the TC life cycle, and therefore the CE rarely existed. We have compared JTWC TC center position with that of JMA and STIPS. The difference between JTWC and JMA center positions is on the average about $10 \mathrm{~km}$. The JTWC center positions are nearly identical to that of the STIPS. Wimmers and Velden (2010) proposed the Automatic Rotational Center Hurricane Eye Retrieval (ARCHER) method to find the center of hurricane. The accuracy of the ARCHER-estimated center position is $9 \mathrm{~km}$ for category 1-5 hurricanes. It appears that $10 \mathrm{~km}$ may be the uncertainty range.

To further smooth the data and also to be consistent with the center position uncertainty of $10 \mathrm{~km}$, we employee five pixel averages in the radial direction and a $45^{\circ}$ sector average of $T_{B}$ to obtain eight radial profiles for the bin data. In each bin of the radial profile, the $T_{B}$ mean value and the standard deviation $\sigma$ within each $45^{\circ}$ sector were calculated. By carefully examining the characteristics of the CE typhoons as in Kuo et al. (2009), an objective method is developed to identify the CE typhoon from the eight radial profiles of $T_{B}$ data. The method involves the following five sequential steps:

1) Within $150-\mathrm{km}$ distance of the TC center, check for the existence of one local maximum $T_{B}$ (weak convection) between two minimum $T_{B}$ (strong convection) in each profile representing the average of each $45^{\circ}$ section.

2) Check in each profile that the local $T_{B}$ maximum and minimum satisfy the criteria of $T_{B_{\max }} \geqq \sigma_{\text {outer_min }}+$ $T_{B_{\text {outer_min }}}$ and $T_{B_{\text {max }}} \geqq \sigma_{\text {inner_min }}+T_{B_{\text {inner_min }}}$.

3) For the radial profiles that satisfy the criteria 1 and 2 , check if $T_{B_{\text {outer_min }}} \leqq 230 \mathrm{~K}$.

4) Check if at least five out of eight sectors/profiles satisfy the above three criteria.

5) Check if the radial distance between any sectors of the two outer eyewalls is smaller than $50 \mathrm{~km}^{2}$

Criterion 1 identifies the existence of the structure that resembles the moat and the double eyewall in each of the eight radial profiles. Criterion 2 ensures that the moat is significant and criterion 3 ensures that the outer eyewall has strong convection (Kuo et al. 2009). Criterion 4 ensures axisymmetry and criterion 5 ensures that the outer eyewall identified is not a spiral band. Figure 1 gives an example of the CE Typhoon Oliwa (1997) and the no-CE Typhoon Vamco (2009) and their associated $T_{B}$ radial profiles. Note that the $T_{B}$ is set to decrease upward so that a low $T_{B}$ value (deep convection) is high in the coordinate. It is clear from Fig. 1 that both typhoons have the $T_{B}$ profiles of two local minima (two eyewalls) and one maximum in between the minima (the moat). The no-CE Typhoon Vamco (2009) is not classified as a CE typhoon based on our criterion 5, with the convection in the outer eyewall identified as a spiral band.

Regarding the axisymmetry consideration, Hawkins and Helveston (2004) used a one-half circle criterion to identify CE typhoons. Our criterion 4 of $5 / 8$ azimuthal coverage is stricter than that of Hawkins and Helveston (2004). If we chose $\% / 8$ profiles for the CE criteria, only 65 CE cases would have been identified. We employ the $5 / 8$ criterion to ensure the CE typhoons identified are axisymmetric with a significant moat and a strong convective outer eyewall while retaining enough cases for statistics. The objective method allows us to systematically identify CE typhoons from a very large pool of data. Table 1 shows the number of SSM/I and TMI images that were processed, recognized CE images, cases and TCs, and repeated CE formation cases. We examined 26774 SSM/I and TMI satellite images from the

\footnotetext{
${ }^{2}$ Following the suggestion of the reviewer, we have also used the criterion that the radial distance between any sectors of the two outer eyewalls is smaller than $25 \%$ of the outer eyewall mean radius. The $25 \%$ criterion is reasonable in that the degree of spiraling out is proportional to the size of the storms. We found that both criteria produce the same results in our study.
} 
(a) $199702 \mathrm{C}$ Oliwa

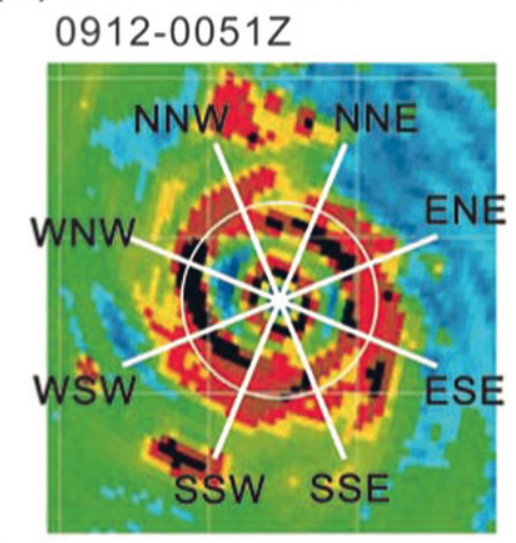

(b) 2009 11W Vamco 0824-2000Z
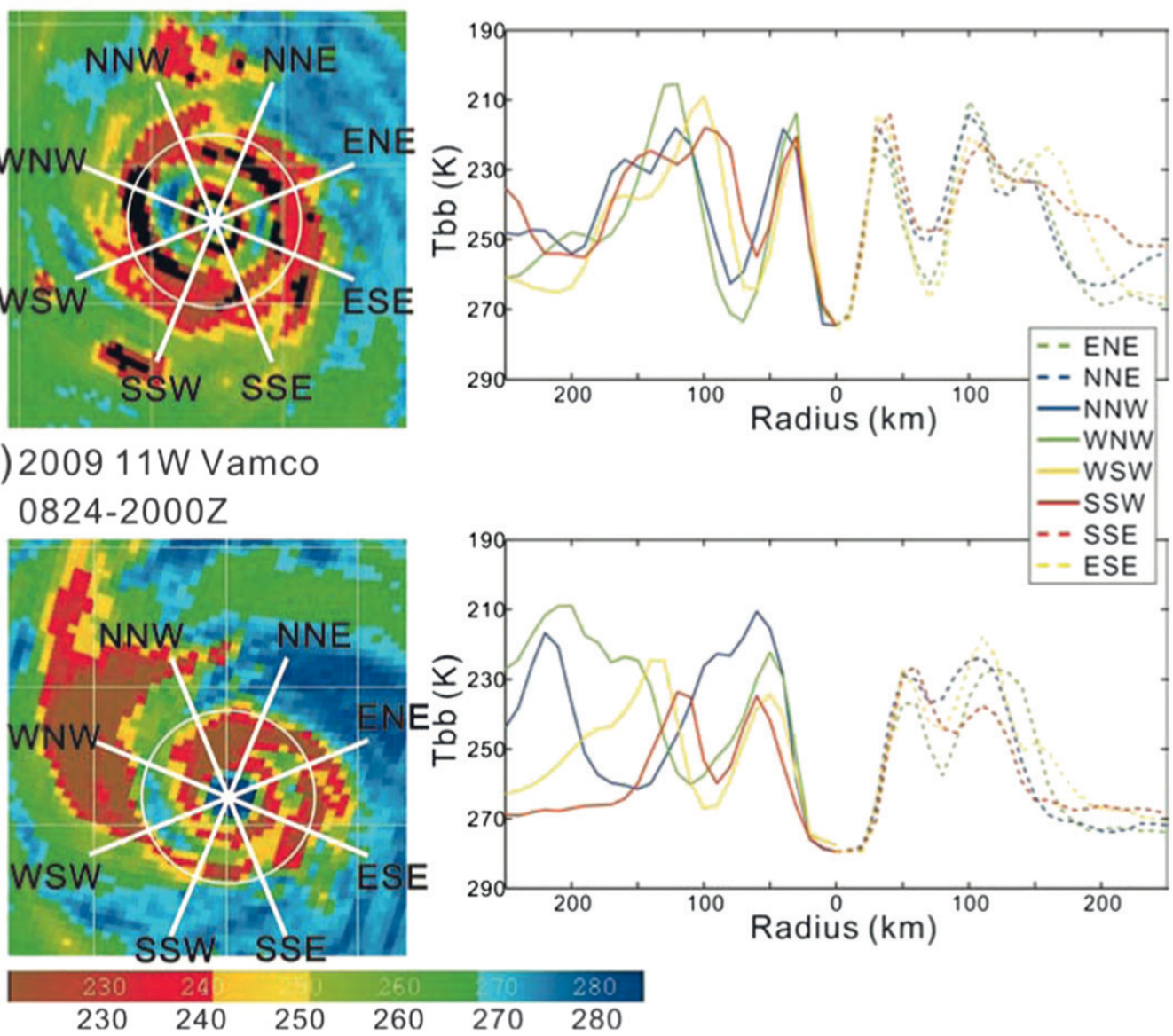

FIG. 1. Color-enhanced microwave CE imageries of Typhoons (a) Oliwa (1997) and (b) Vamco (2009). The averaged $T_{B}$ profiles of eight radial directions for Typhoon Oliwa are conformed to the CE-determined criteria. The secondary $T_{B}$ minimum for Typhoon Vamco only identified spiral outer rainband. (solid green: WNW, solid yellow: WSW, solid red: SSW, solid blue: NNW, dash green: ENE, dash yellow: ESE, dash red: SSE, and dash blue: NNE).

NRL website. Out of these, $95 \mathrm{CE}$ cases with $234 \mathrm{CE}$ images were identified, including 16 cases of multiple CE formation. There are $77 \mathrm{CE}$ typhoons identified. The CE structure exists in approximately $35 \%(64 \%)$ of the typhoons with category 1 (category 4 ) or greater intensity from 1997 to 2011 in the western North Pacific basin. Note that the objective method misses one case (Typhoon Tina 1997) that would have been identified using subjective identification of Kuo et al. (2009). The rejection of the case as CE is because of the $230-\mathrm{K}$ outer eyewall criterion.

Kuo et al. (2000) studied the merger of tropical cyclones Zeb and Alex. Their analysis based on GMS IR image is from 1800 UTC 9 October to 1800 UTC 12 October. The observed merger processes appear to be in good agreement with the straining out regime of Dritschel and Waugh (1992), in that Alex was quickly elongated and wrapped cyclonically around Zeb to become a spiral band of Zeb. Our analysis indicated that, shortly after 1800 UTC 12 October, a CE structure of Typhoon Zeb possessed a CE structure at 2234 UTC 12 October 1998 (not shown). This case provides an example of CE formation by binary TC vortex interaction (Kuo et al. 2004).

TABLE 1. Numbers of SSM/I images, TMI images, recognized $\mathrm{CE}$ images and cases, TC, and repeated CE formation cases obtained from on the NRL website.

\begin{tabular}{|c|c|c|c|c|c|c|}
\hline Total & Images & $\begin{array}{c}\mathrm{CE} \\
\text { images }\end{array}$ & $\begin{array}{l}\mathrm{CE} \\
\text { cases }\end{array}$ & $\mathrm{TC}$ & \multicolumn{2}{|c|}{$\begin{array}{l}\text { Repeated CE } \\
\text { formation cases }\end{array}$} \\
\hline SSM/I & 16431 & 234 & 95 & 77 & Twice & 14 \\
\hline TMI & 10343 & & & & 3 times & 2 \\
\hline
\end{tabular}


The results indicated that two TC of different sizes were with mutual cyclonic rotation, followed by the complete straining out of Alex into a spiral band of Zeb and finally the formation of CE structure in Zeb.

To further quantify our results, the inner eyewall radius was defined as the distance between the typhoon center to the point where $T_{B}=0.5 \times \sigma_{\text {inner }}+T_{B_{\text {inner }}}$. The moat width was defined by the distance between the points of $T_{B} \geqq 0.5 \times \sigma_{\text {outer }}+T_{B_{\text {outer }}}$ and $T_{B} \geqq 0.5 \times$ $\sigma_{\text {inner }}+T_{B_{\text {inner }}}$. Finally, the outer eyewall width was defined by the distance of the region that satisfies $T_{B}<$ $0.5 \times \sigma_{\text {outer }}+T_{B_{\text {outer }}}$ in the outer eyewall region. The inner eyewall radius, the moat width, and the outer eyewall width were calculated by averaging the radial profiles of the eight azimuthal sections shown in Fig. 1.

\section{Intensity and structural changes}

To study the structural and intensity changes of $\mathrm{CE}$ typhoons, 25 cases were excluded when the typhoon center was within $200 \mathrm{~km}$ from land in the period of $24 \mathrm{~h}$ before and after $\mathrm{CE}$ formation, or where the satellite temporal resolution was greater than $12 \mathrm{~h}$. There were $70 \mathrm{CE}$ cases analyzed. Following the work of Hawkins and Helveston (2008), three different structural change processes were defined after CE formation. The ERC cases were classified based upon the dissipation of the inner eyewall in less than $20 \mathrm{~h}$ after CE formation. The ERCs repeated multiple times classification is treated as separate CE case here. The cases in which part of the outer eyewall dissipates within $20 \mathrm{~h}$ are classified as no replacement cycle (NRC) cases. The cases where the CE structure is maintained for more than $20 \mathrm{~h}$ (and the inner cores in the sequence of imagery have a similar size) are classified as concentric eyewall maintained (CEM) cases. The similar inner core size requirement was used to avoid assigning a CE typhoon with multiple ERC processes into one single CEM case.

Sitkowski et al. (2011) indicated the microwave imagery CE structure is visible nearly $18 \mathrm{~h}$ after the $\mathrm{CE}$ structure is first observed in the aircraft observations. Because the observed replacement cycle is $36 \mathrm{~h}$, and the CE structure may simultaneously disappeared both in the aircraft and microwave data, the ERC time scale is about $18 \mathrm{~h}$. Thus, the 20 -h criterion is in accordance with observations of Sitkowski et al. (2011). Moreover, the criterion is larger than the characteristic time of ERC from the observations (Kuo et al. 2004; Willoughby et al. 1982; Willoughby and Black 1996) and from numerical modelings (Terwey and Montgomery 2008; Qiu et al. 2010; Zhou and Wang 2009).

Knaff et al. (2003) suggested that annular hurricane (AH) formation is preceded by a significant asymmetric mixing event in which possible mesovortices mix eyewall air into the eye and vice versa. Zhou and Wang (2009) presented model simulations of CE lead to the formation of $\mathrm{AH}$ in $6 \mathrm{~h}$. We have also examined the possibility of $\mathrm{AH}$ in our dataset. We do not have a definite conclusion because of the lack of high-temporalresolution satellite data. On the other hand, our identification of CEM and NRC cases will not be affected by the possible AH pathway in ERC. We will incorporate the AMSR-E and SSM/IS data for better temporal resolution to study this mechanism in the future.

The ERC classification had 37 out of 70 cases $(53 \%)$. The CEM and NRC classification had 16 (23\%) and 17 cases $(24 \%)$. Examples of the three classifications for the CE processes are shown in Fig. 2. In addition to the $T_{B}$ images, two half-plane averaged $T_{B}$ radial profiles are shown with respect to the center of the storm. Of all the possible four pairs of two half planes from the eight radial profiles, the average profiles shown in Fig. 2 are the pair with the greatest asymmetries. The $T_{B}$ profiles indicate that Typhoon Saomai (2000) is an ERC case, with the inner core dissipated approximately $12 \mathrm{~h}$ after CE formation. Typhoons Haitang (2005) and Ewiniar (2006) are NRC cases, where the inner eyewall was barely maintained while the outer eyewall disappeared appreciably into a half circle. This NRC cases are similar to "the shear stop ERC mode" classified by Hawkins and Helveston (2008). The dissipation of the outer eyewall may be related to an environmental factor such as the vertical shear. This is examined further in section 4. Typhoons Winnie (1997), Dianmu (2004), and Chaba (2004) are CEM cases that maintain their CE structure for approximately $36 \mathrm{~h}$. These cases have large moat widths $(137,45$, and $70 \mathrm{~km}$, respectively) and large outer eyewall widths $(137,64$, and $85 \mathrm{~km}$, respectively). The CEM cases resemble "the large radius outer eyewall and CE structure maintained for a time cases" of Hawkins and Helveston (2008). Note that there is significant asymmetric convection outside of the inner core $12 \mathrm{~h}$ before CE formation in most of the examples in Fig. 2. Kuo et al. (2004) also noted asymmetric convection outside the core of Typhoon Lekima (2001). Figure 3 shows the composite time series of (Fig. 3a) strong convection percentage $\left(T_{B} \leqq 230 \mathrm{~K}\right)$ and (Fig. 3b) weak convection percentage $\left(230<T_{B} \leqq 270 \mathrm{~K}\right)$ for ERC, CEM, NRC, and for the NCE cases (no-CE typhoons with intensity of category 4 or greater). ${ }^{3}$ The composite was made relative to the $\mathrm{CE}$ formation time or the peak

\footnotetext{
${ }^{3}$ Excluding the cases where TC centers were within $200 \mathrm{~km}$ close to land in the time period of $48 \mathrm{~h}$ before and after the peak intensity.
} 
(a)

2000-22W

Saomal

ERC

a.0909-1129Z

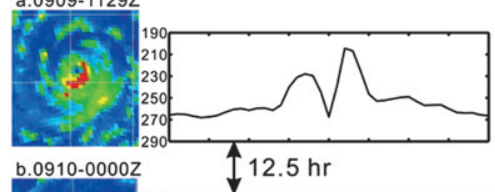

b. $0910-0000 z$

$\sum_{-3}(x)=2$

$\frac{\text { c.0910-1117z }}{\text { maot width: } 35 \mathrm{~km}}$

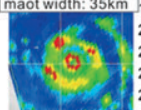

d.0910-2347z
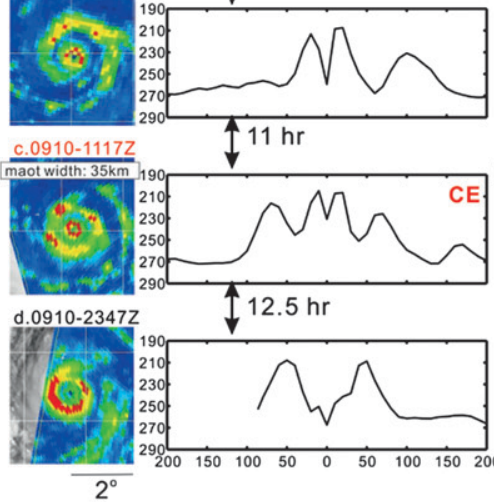

$\uparrow 12.5 \mathrm{hr}$

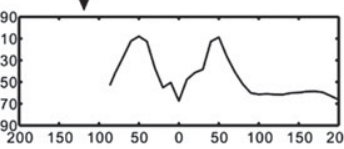

(d)

1997-14W

Winnie

CEM

a.0814-2316z

(1) 8$)$

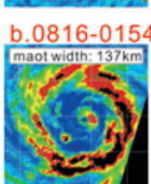

c. $0816-1248$

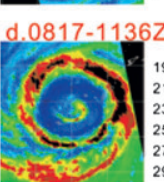

e. 0818-0021

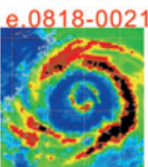

f.0818-1123

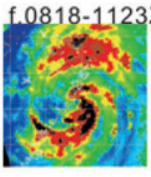

$\overline{2^{\circ}}$

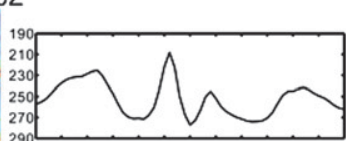

$4 \mathrm{\downarrow} \ 26.5 \mathrm{hr}$

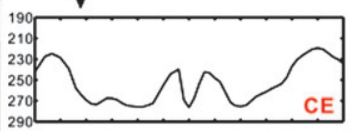

$\uparrow 11 \mathrm{hr}$
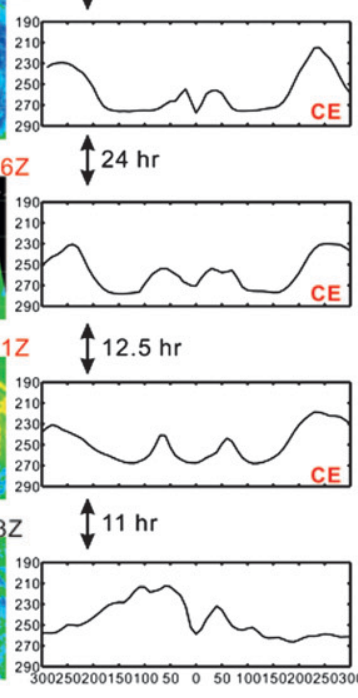

(b)

2005-05W

Haitang

NRC

a.0715-0959z

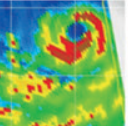

b. $0715-2231 Z$
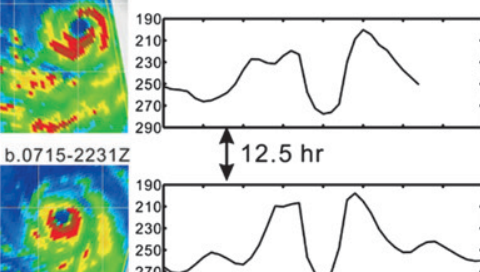

$\downarrow_{12.5} \mathrm{hr}$

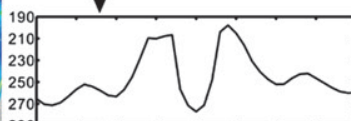

c. $0716-09452$
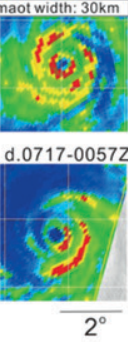

(e)

2004-09W

Dianmu

CEM

a.0617-1008z
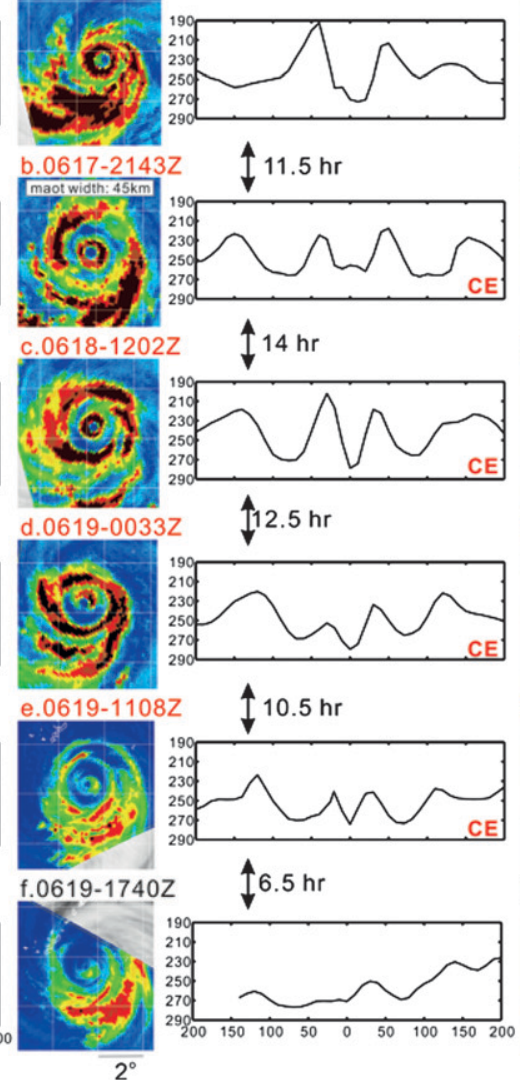

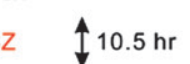

(c)

2006-04W

Ewiniar

NRC

a. 0706-1528z
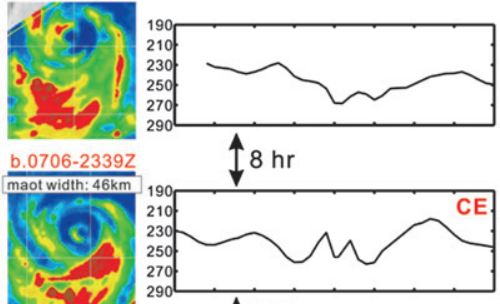

† $5.5 \mathrm{hr}$
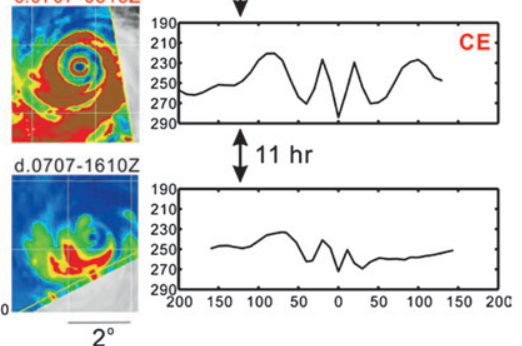

(f)

2004-19W

Chaba

CEM

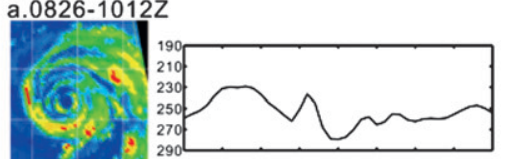

b. 0826-2203z
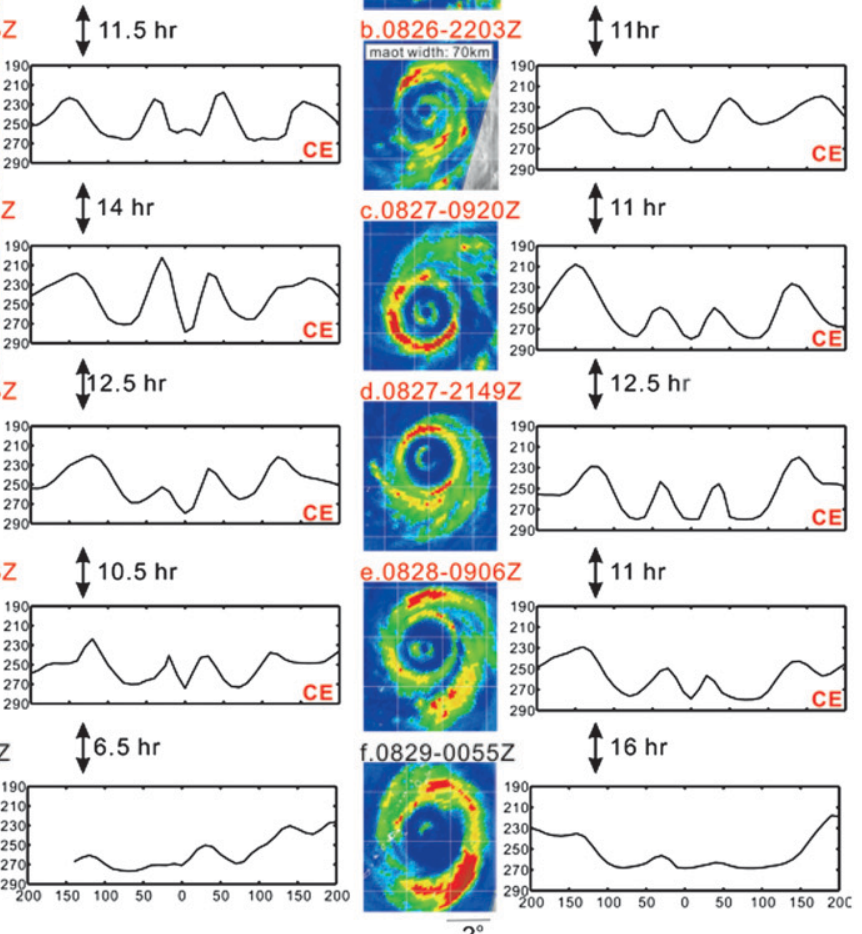

FIG. 2. The imagery sequences and averaged $T_{B}$ radial profile for (a) Typhoon Saomai 2000-ERC, (b) Haitang 2005-NRC, (c) Ewiniar 2006-NRC, (d) Winnie 1997-CEM, (e) Dianmu 2004-CEM, and (f) Chaba 2004-CEM. 
(a)

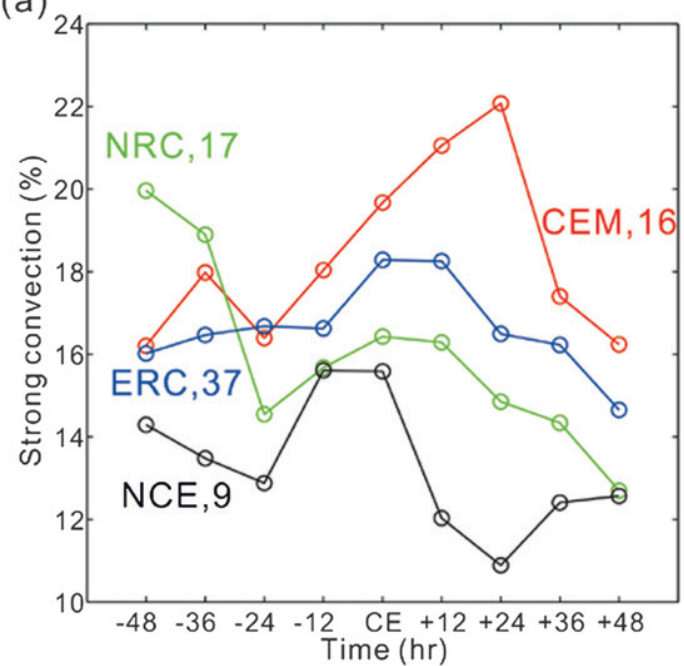

(b)

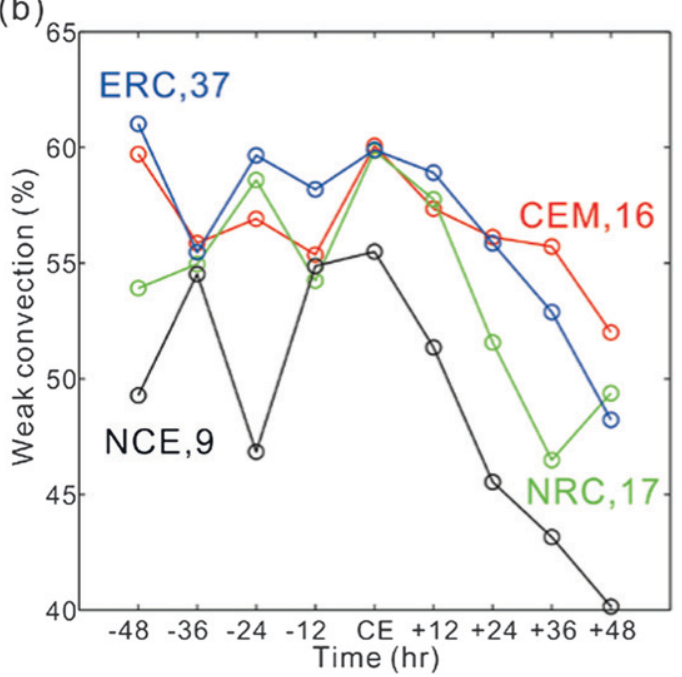

FIG. 3. Composite time series of the percentage for (a) strong convection $\left(T_{B} \leqq 230 \mathrm{~K}\right)$ and (b) weak convection $(230<$ $T_{B} \leqq 270 \mathrm{~K}$ ) for ERC (blue), CEM (red), NRC (green), and NCE (black). The composite was done relative to the time of $\mathrm{CE}$ formation (maximum intensity) for the $\mathrm{CE}$ (NCE) cases. The numbers of cases for each category are indicated.

intensity time. Figure 3 indicates that the areal percentage of strong convection is largest for the CEM cases, followed by the ERC, NRC, and NCE cases. The overall areal coverage of weak convection is greater than that of the strong convection. Figure 3 indicates that the strong and weak convection all decrease after CE formation (peak intensity) in the NRC (NCE) cases. The areal coverage of weak convection also decreases after $\mathrm{CE}$ formation for the ERC and CEM cases (Fig. 3b). On the other hand, the deep convection increases for $24 \mathrm{~h}$
(CEM) and is maintained for $12 \mathrm{~h}$ (ERC) after CE formation (Fig. 3a). We also test the no-CE typhoons with intensity of categories $1-3$. The result is similar to NCE cases except that the range of the scale is reduced.

Figure 4 shows the composite time series of intensities and normalized intensity for the ERC, CEM, and NRC cases as well as the average of the total CE sample. The normalized intensity of Emanuel (2000) and Knaff et al. (2003) are plotted for the comparison. The composite
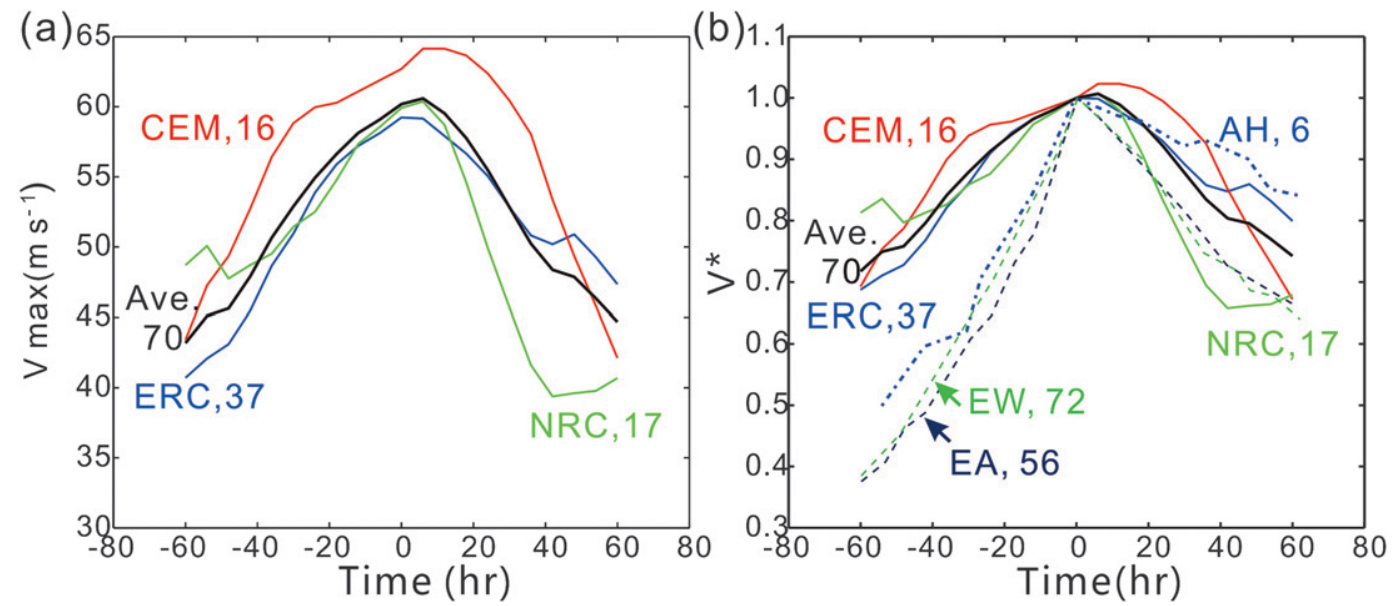

FIG. 4. Composite time series of (a) intensity and (b) normalized intensity for the ERC, CEM, NRC cases, and the all CE cases. The normalized intensities associated with average tropical cyclones in the Atlantic (EA) and western North Pacific (EW) that did not encounter cold water or make landfall as reported by Emanuel (2000), and annular hurricanes (KK) as reported by Knaff et al. (2003) are plotted for comparison. The composite was computed relative to $\mathrm{CE}$ formation time (for all CE cases), annular hurricane formation time (for Knaff et al.'s cases), or the maximum intensity time (for NCE cases and Emanuel's cases). The number of cases is indicated next to each line. 
was computed relative to the $\mathrm{CE}$ formation time (for all CE cases), annular hurricane formation time (for Knaff et al. 2003 cases), or the maximum intensity time (for NCE cases and Emanuel's cases). Figure 4a suggests that the average intensity of CEM cases is stronger than that of the ERC and NRC cases before and after CE formation. In particular, the CEM storms intensified continuously for $18 \mathrm{~h}$ after CE formation and maintained the intensity for another $24 \mathrm{~h}$. The composite intensity of ERC and NRC cases are similar before CE formation. However, the intensity of NRC decreases appreciably after $\mathrm{CE}$ formation. It is interesting to note that both the ERC and NRC cases reintensified approximately $48 \mathrm{~h}$ after CE formation. The ERC cases intensity change may be related to the outer eyewall contracting inward while it conserves angular momentum. The NRC cases are related to the vertical wind shear reduction (not shown). Figure $4 \mathrm{~b}$ indicates that a key feature of $\mathrm{CE}$ formation appears to be the maintenance of a relatively high intensity for a longer duration, rather than a rapid intensification process to a high intensity. The stronger core intensity may play a pivotal role in the axisymmetrization dynamics of asymmetric convection outside the core to produce the CE structure (Kuo et al. 2004). Hence and Houze (2012) studied the vertical structure of CE hurricane from TRMM precipitation radar. Their results suggest that secondary eyewalls form from low-level rainbands that undergo axisymmetrization. Knaff et al. (2003) showed that AH tend to experience a long period of steady intensity with a relatively slowly decreasing intensity. Figure $4 \mathrm{~b}$ suggests that, on the average, the intensity for all the $\mathrm{CE}$ cases tends to decrease more slowly than that of the TCs identified in Emanuel (2000) in the first $18 \mathrm{~h}$ after $\mathrm{CE}$ formation, and the intensity for the CEM cases decreases at a rate that is slower than that of the annular cases (Knaff et al. 2003) in the $36 \mathrm{~h}$ after CE formation. Our results suggest that the CEM cases are CE typhoons with higher intensity and more convective activity (CA) for a longer period of time.

Maclay et al. (2008) use aircraft data to construct the $K-$ Vmax diagram for the intensity and structural changes. The aircraft reconnaissance data used to calculate the areal integrated kinetic energy in the $K-$ Vmax diagram is not available in the western North Pacific basin. Therefore, the structural and intensity variability is illustrated here using the $T$-Vmax diagram (where $T$ is the $T_{B}$ and Vmax is the best-track estimated intensity), so that a time sequence of intensities and CA can be shown. The CA is indicated by the areal averaged $T_{B}$ contrast to the background $T_{B}$ in the $400 \mathrm{~km}^{2}$ area of satellite imagery centered at the eye $\left(\mathrm{CA} \equiv-\overline{T_{\mathrm{B} 1}-T_{\mathrm{B} 0}}\right)$. The background $T_{\mathrm{B} 0}$ is calculated as the highest $5 \%$ of $T_{B}$ in the $400 \mathrm{~km}^{2}$ area. The $400 \mathrm{~km}^{2}$ box in general is sufficient to cover the structure of $\mathrm{CE}$ typhoons. ${ }^{4} \mathrm{~A}$ lower value of the areal averaged $T_{B}$ implies stronger CA. Our analysis suggests that a decrease in the areal averaged $T_{B}$ and increase in CA is often associated with an increase of the deep convection area $\left(T_{B} \leqq 230 \mathrm{~K}\right)$.

Figure 5 shows the $T$-Vmax diagram for the no-CE Typhoon Rammasun (2008), the ERC Typhoon Babs (1998), the CEM Typhoon Maemi (2003), and the NRC Typhoon Shanshan (2006). The no-CE Typhoon Rammasun is at category 4 intensity over the ocean far from the land. Figure 5 indicates that both the intensity and CA of Typhoon Rammasun increased before it reached the maximum intensity $\left[135 \mathrm{kt}\left(\sim 69.4 \mathrm{~m} \mathrm{~s}^{-1}\right)\right]$ in its lifetime; the maximum intensity was maintained for a period of time ( $12 \mathrm{~h}$ ) while CA decreased, and then both the intensity and CA decreased after approximately $36 \mathrm{~h}$. The intensity and CA decrease in the 12-h period after CE formation in the NRC Typhoon Shanshan. On the other hand, the intensities of ERC Typhoon Babs and CEM Typhoon Maemi decreased after CE formation, even though their CA remained steady or increased slightly. The maintenance or a slight increase of the CA for the CEM and ERC cases is in general agreement with the notion that the CE typhoon can lead to storm growth (Maclay et al. 2008). The decrease of areal averaged $T_{B}$ (due to the increase of deep convection) and the increase of kinetic energy both occurred after the ERC process.

Figure 6 shows the $T$-Vmax diagrams for average values of intensity and CA for the NCE typhoons (9 cases), ERC typhoons (37 cases), CEM typhoons (16 cases), and NRC typhoons (17 cases). The time period plotted in Fig. 6 is $48 \mathrm{~h}$ before and after the peak intensity/ $\mathrm{CE}$ formation. Figure 6 indicates that the time sequence of the average Vmax and CA (in terms of $T_{B}$ contrast) is similar to that in Fig. 5. On average, the CEM and the ERC cases have stronger CA after CE formation. In particular, the average for the CEM cases indicates significant $\mathrm{CA}$ increase $24 \mathrm{~h}$ after $\mathrm{CE}$ formation. We also test the no-CE typhoons with intensity of categories 1-3. The result is similar to NCE cases except the range of $T_{B}$ and Vmax variations are reduced.

\section{Structure characteristics and environmental factors}

To further explore the characteristics of moat width in CE typhoons, scatterplots of the moat width and outer

\footnotetext{
${ }^{4}$ Typhoons Winnie (1997) and Amber (1997) were very large. These quantities are calculated using a $600 \mathrm{~km}^{2}$ box.
} 

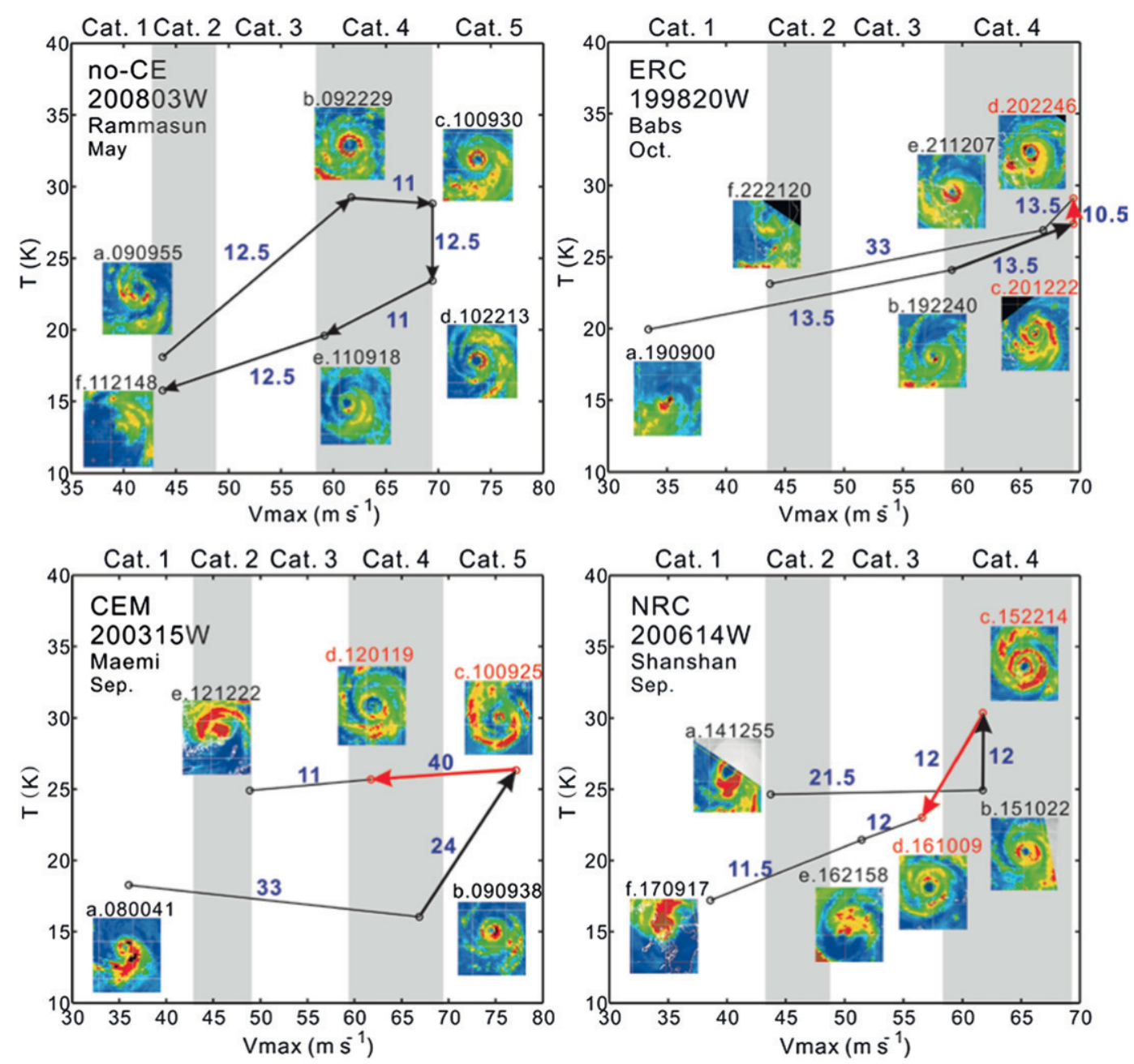

FIG. 5. T-Vmax diagrams for no-CE Typhoon Rammasun (2008), ERC Typhoon Babs (1998), CEM Typhoon Maemi (2003), and NRC Typhoon Shanshan (2006); $T$ is the difference between the background $T_{B}$ and averaged $T_{B}$ for each image within $400 \times 400 \mathrm{~km}^{2}$ centered at the storm. The blue numbers indicate time differences (h) between satellite imageries. The black (red) arrow indicates the averaged $T_{B}$ and intensity changes before (during) CE period. The averaged $T_{B}$ and intensity change in ERC (NRC) cases is the difference between the first ERC (NRC) and $\mathrm{CE}$ formation imageries. The change in CEM cases is the difference between the last CE maintained and CE formation imageries.

eyewall width, and the moat width and intensity are shown in Fig. 7. We also include the average values of moat width, outer eyewall width, and intensity in the figure. Figure 7 indicates that the outer eyewall width is larger with a larger moat width $\left(R^{2}=0.5\right)$. All the CEM cases have moat widths greater than $40 \mathrm{~km}$. In particular, the CEM cases on average have slightly higher intensities $\left(63 \mathrm{~m} \mathrm{~s}^{-1}\right)$, larger moat widths $(61 \mathrm{~km})$, and larger outer eyewall widths $(70 \mathrm{~km})$ than that of ERC and NRC cases. The average moat width of the CEM case is about $20 \mathrm{~km}$ larger than that of the NRC and ERC cases. Even when excluding Typhoon Winnie (1997), which had an exceptional 138-km moat width in our CEM cases, the average moat width of CEM cases is still 12 (14) $\mathrm{km}$ wider than that of the NRC (ERC) cases. The four CEM cases with weaker intensities of categories 2 and 3 in Fig. 7 all have both very large moat width (larger than $60 \mathrm{~km}$ ) and outer eyewall width (larger than $80 \mathrm{~km}$ ). In general, the moat size and outer eyewall width are approximately $50 \%$ larger in the CEM cases than that in the ERC and NRC cases.

The very large moat and outer eyewall width in the CEM cases may have some implications for the long duration of CE structure. Willoughby (1979) presented a scale analysis on the validity of the balance model and the transverse circulation equation in the TC. Rozoff et al. (2008) used the balanced model transverse circulation equation to study the ERC dynamics. In this 

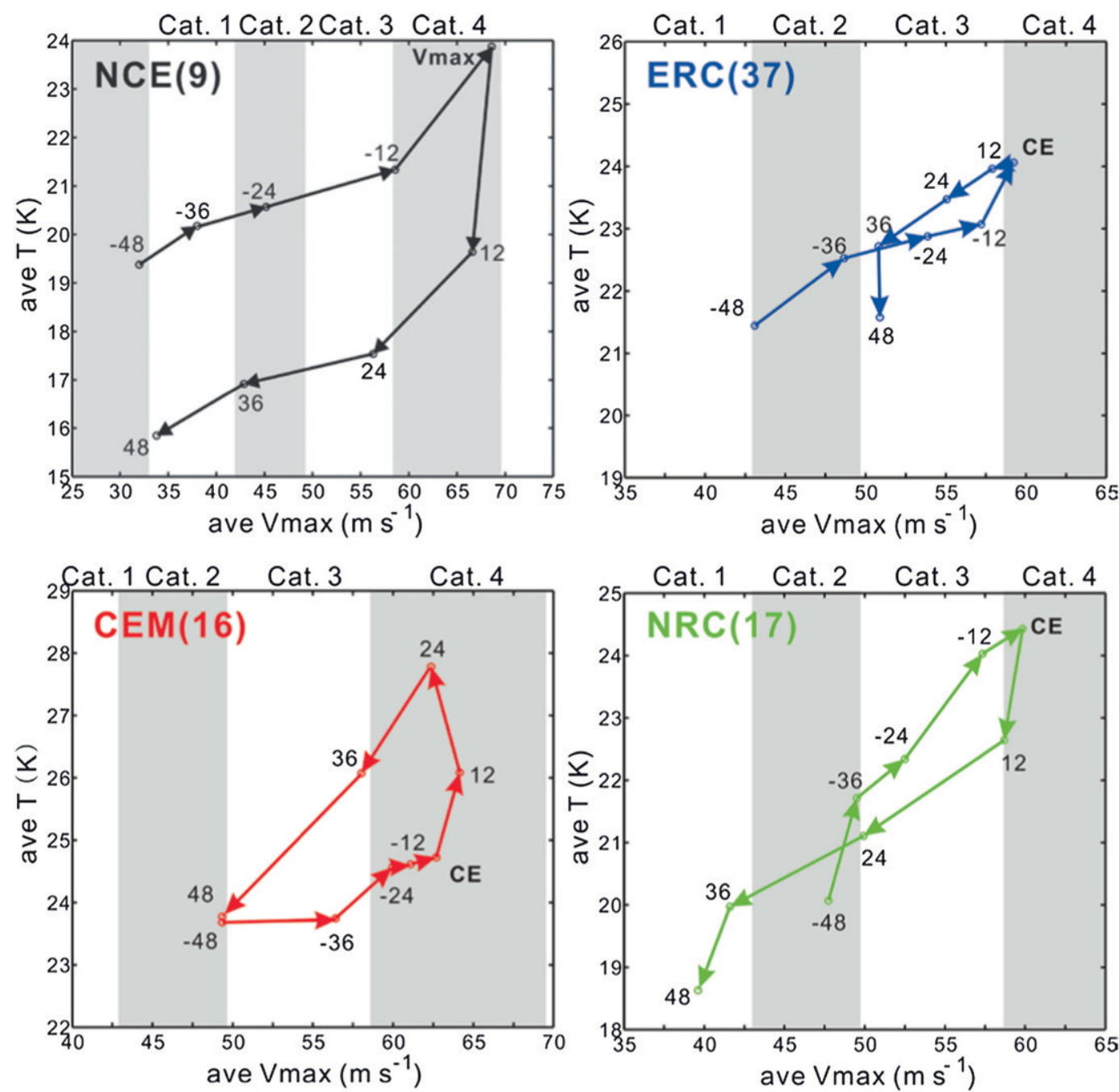

FIG. 6. The averaged $T_{B}$ and intensity changes in $48 \mathrm{~h}$ before and after the peak intensity/CE formation for the NCE, ERC, CEM, and NRC cases. Case numbers are given in parentheses. Note that the plotting scale is slightly different in each case.

manner, the balance dynamics of the CE is scale free; namely, the dynamics may occur in different scales where the balance equation assumption is valid. Thus, it is possible that the larger CE storms simply end up taking much longer time to contract because of their larger scale. Rozoff et al. (2008) showed that the decay of the inner eyewall may be related to the fact that the upper warm core has a larger stabilization effect on the convection in the inner eyewall than it does on the convection in the outer eyewall. The stabilization effect of upper warm core argument cannot explain why the inner eyewall is maintained for such a long time in the CEM cases.

On the other hand, the occurrence of barotropic instability (e.g., Kossin et al. 2000) will invalidate the axisymmetric balance assumption. Kossin et al. (2000) identified two types of barotropic instabilities in the vorticity field with $\mathrm{CE}$ structure; the instabilities across the outer eyewall (the type I) and across the moat (the type II) due to the sign reversal of the radial vorticity gradient (i.e., the Rayleigh necessary condition). These instabilities may work against the maintenance of the CE structure. The large moat size in the CEM cases has two dynamic implications. It reduces the growth rate of the type II instability across the moat which is favorable for the CE structure maintenance; and it also lessens the stabilization of the core vortex on the type I instability across the outer eyewall (i.e., the Fjørtoft sufficient condition), which is not favorable for the CE maintenance. As demonstrated by Kossin et al. (2000), the thicker outer eyewall is more stable for the type I instability, which is favorable for maintaining the outer eyewall 


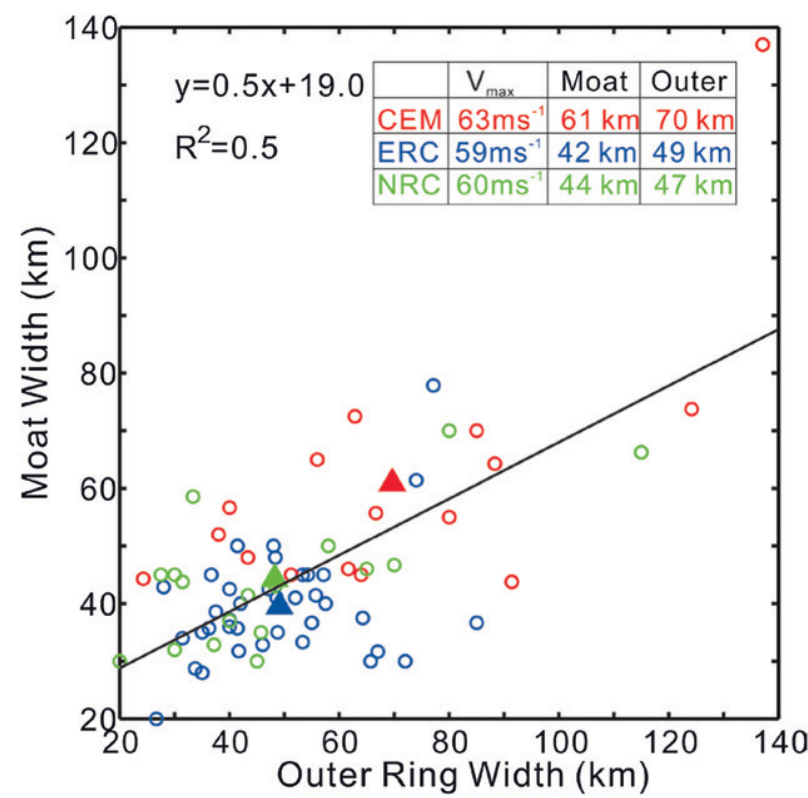

FIG. 7. Scatter diagram of the moat width vs outer eyewall width. The linear fitting line and formula are also shown. The table indicates the average Vmax, moat width, and outer eyewall width of CEM, ERC, and NRC cases, respectively.

structure. These observations of the large outer eyewall and moat widths are in general agreement with the concept that barotropic dynamics may play a significant role in maintaining the $\mathrm{CE}$ structure for CEM cases.

Finally, we note that the large moat size in the CEM cases may have an impact on the convection and subsidence in both eyewalls. The interference between the convection/subsidence couplet of the inner and outer eyewalls may be reduced when the moat size is very large. The large moat size may assist the inner core by suppressing potentially competing convection while the subsidence concentrated radial outward may make it less likely to penetrate to the eyewall. Zhou and Wang (2011), in the modeling study, revealed the demise of the inner eyewall is primarily due to the interception of the boundary layer inflow supply of entropy by the outer eyewall. The interception process becomes inefficient when the moat size is large. Since the CEM cases are with long duration of significant storm size, the dynamics of large moat CEM cases deserves closer investigation.

Rozoff et al. (2006) proposed that a rapid filamentation zone outside the radius of maximum wind in strong TCs may play a role, in addition to the subsidence, on the moat formation in the CE TC. The rapid filamentation zone is a region of strain-dominated flow where the filamentation time is shorter than the typical moist convective overturning time of $30 \mathrm{~min}$. The nondimensional filamentation width in the axisymmetric model is $d_{\mathrm{ft}} / r_{0}=$ $\left[\left(\sqrt{\alpha} \zeta_{0} \tau_{\text {con }}\right)^{1 / 1+\alpha}-1\right]$, where $r_{0}$ is the satellite estimated core size; $\zeta_{0}$ is the core vorticity strength estimated by JTWC intensity $r_{0} ; \tau_{\text {con }}$ is the convective overturning time of $30 \mathrm{~min}$; and $\alpha$ is the vorticity skirted parameter. The derivation of the nondimensional formula is given in Kuo et al. (2009). The typical vorticity skirted parameter is $0.5 \leq \alpha \leq 1$. We find our results are insensitive to the choice of $\alpha$.

Figure 8 gives the variance $R^{2}$ of the observed nondimensional moat width that is explained by the nondimensional filamentation width for all CE, CEM, ERC, and NRC typhoons. The nondimensional width calculation is done according to the core size $r_{0}$ from the satellite observations. Figure 8 indicates that the filamentation moat width explains more than $55 \%$ of the variance of the satellite observed moat width. In particular, the CEM (NRC) cases are explained by $54 \%(60 \%)$ and the filamentation dynamics appear to be relevant to the moat size variation. This result does not suggest that subsidence is not important in the maintenance of the CE typhoon moat; rather, it may be that in a strong TC the subsidence is confined to the edge of the deep convection by inertial stability and is therefore less likely to be uniform in the moat region (analogous to the eye subsidence distribution study of Schubert et al. 2007). It indicates that vortex scale filamentation dynamics lead to the organization of moat size. Kuo et al. (2012) also found that the vortex-scale filamentation dynamics may be important in suppressing deep convection and organizing spiral bands in the TC environment. Figures 7 and 8 suggest that the internal structure of CE typhoons, such as the general high intensity with the large widths of the moat and outer eyewall, may be important for the maintenance of the CE structure in the CEM cases.

Figure 9 shows the locations of $\mathrm{CE}$ formation on the tracks $24 \mathrm{~h}$ before and after the formation for the CEM (red), ERC (blue), and NRC (green) cases. The two triangle symbols are the average positions at the time of $\mathrm{CE}$ formation and $24 \mathrm{~h}$ afterward, respectively. Most $\mathrm{CEM}$ cases are located west of $140^{\circ} \mathrm{E}$ with the smallest northward translation speed of $2.9 \mathrm{~m} \mathrm{~s}^{-1}$ (Table 2). This is in general agreement with Fig. 8 in that typhoons tend to be more intense in the western part of Pacific after a long journey over ocean. On the other hand, the NRC cases have a larger northward translation speed of $4.8 \mathrm{~m} \mathrm{~s}^{-1}$. Even though most NRC cases start in the latitudes farther north (north of $18^{\circ} \mathrm{N}$ ) than that of the CEM and ERC cases, they are farther separated in latitudinal distance because of the fast northward translation speed of NRC cases. Typically $24 \mathrm{~h}$ after CE formation, the NRC cases are in the high latitudes, where the vertical shear is larger and the SST is lower.

The composite time series with respect to CE formation time for the SST, 850-200-hPa vertical shear, and 

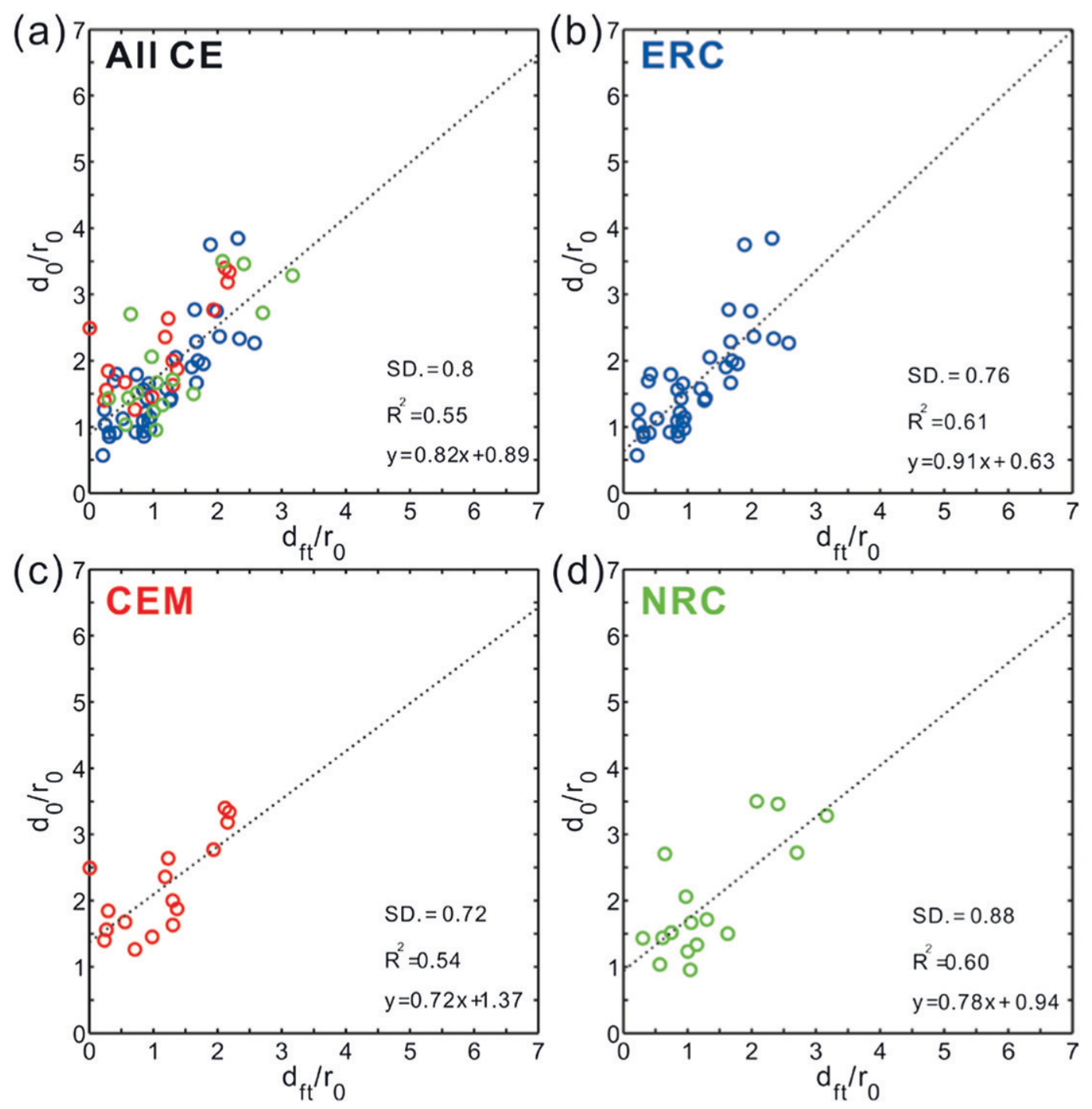

FIG. 8. Scatter diagram of the nondimensional moat width $\left(d_{0} / r_{0}\right)$ vs the nondimensional filamentation moat width $\left(d_{\mathrm{ft}} / r_{0}\right)$ for (a) all CE cases, (b) ERC cases, (c) CEM cases, and (d) NRC cases. The blue, green, and red circles represent individual ERC, NRC, and CEM cases. The $d_{0}, r_{0}$, and $d_{\mathrm{ft}}$ are moat width, inner eye radius, and the width of the rapid filamentation zone, respectively.

850-700- and 700-500-hPa RH, OHC, and the MPI (Emanuel 1997) from the STIPS data are shown in Fig. 10. The standard deviations (SDs) are not plotted for presentation clarity. ${ }^{5}$ On average, the SST, RH, OHC, and MPI decrease and vertical shear increases with time

\footnotetext{
${ }^{5}$ The SDs of SST, vertical shear, OHC, and MPI are small (approximately $0.5^{\circ} \mathrm{C}, 2 \mathrm{~m} \mathrm{~s}^{-1}, 22 \mathrm{~kJ} \mathrm{~cm}^{-2}$, and $4 \mathrm{~m} \mathrm{~s}^{-1}$ ) in the CEM cases throughout the period. In contrast, the SDs in the NRC cases increase with time, with approximately $2^{\circ} \mathrm{C}, 5 \mathrm{~m} \mathrm{~s}^{-1}, 38 \mathrm{~kJ} \mathrm{~cm}^{-2}$, and $22 \mathrm{~m} \mathrm{~s}^{-1}$ values at $24 \mathrm{~h}$ after the CE formation. The SDs of the ERC cases are slightly smaller than that of the NRC cases. The SDs of $\mathrm{RH}$ are similar (approximately $6 \%$ ) in all cases.
}

because typhoons in general move toward the northwest direction. The large northern translation speed of the NRC cases causes them to experience colder SST, larger vertical wind shear, smaller RH, smaller OHC, and smaller MPI $24 \mathrm{~h}$ after $\mathrm{CE}$ formation. The large vertical shear, cool SST, low $\mathrm{RH}$, and small $\mathrm{OHC}$ are consistent with the sharp decrease of CA and intensity in NRC cases. The dissipation of the outer eyewall in the NRC cases presumably may also be related to the strong vertical shear in the high latitudes (Hawkins and Helveston 2008). On the other hand, the CEM cases were under small vertical wind shear; high SST, OHC, and MPI; and high $\mathrm{RH}$ throughout the period of $\mathrm{CE}$ formation. These 


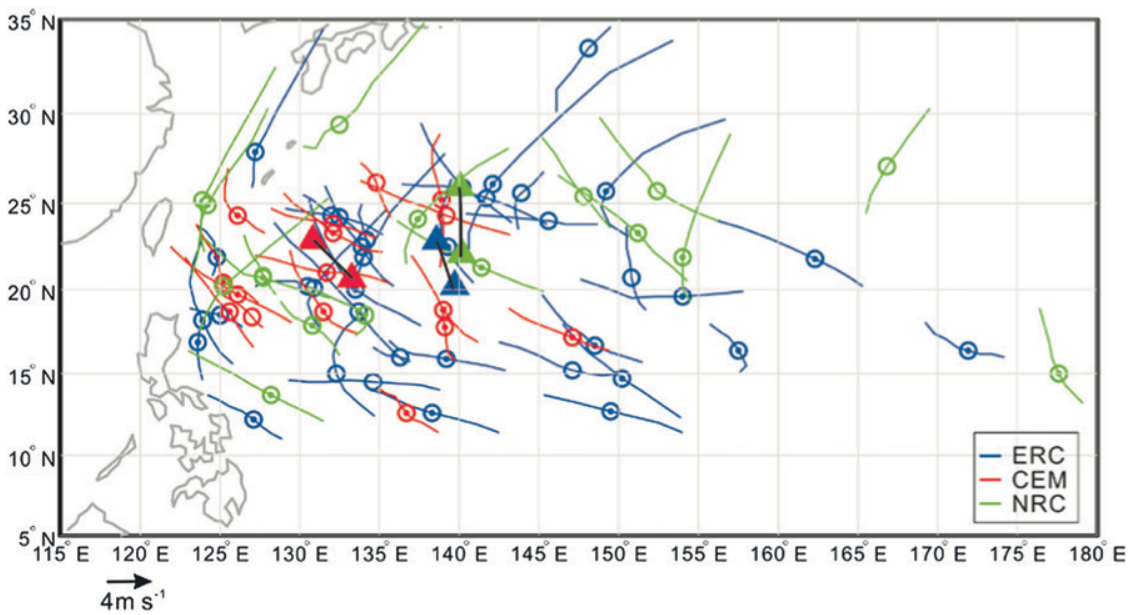

FIG. 9. Tracks within 48-h centered at CE formation in the western North Pacific from 1997 to 2010. The ERC, NRC, and CEM cases are represented by blue, green, and red colors, respectively. The circles with (without) a dot are the location of the secondary eyewall formation with intensity greater than or equal to (less than) category 4 on the Saffir-Simpson scale. The triangle symbols represent the composite location of $\mathrm{CE}$ formation and $24 \mathrm{~h}$ after $\mathrm{CE}$ formation.

favorable environment factors may help CEM cases maintain their intensity and eyewall structures. It is interesting to note that favorable environmental conditions are conductive to the formation and maintenance of the initial (primary) eyewall in Atlantic TC (Vigh et al. 2012). Figure 10 suggests that the environmental conditions play a role in the structural and intensity changes of CEM and NRC typhoons.

Kossin and Sitkowski (2009) stated that the environment plays a significant additional role in modulating the ERC process. Our results agree with this statement as shown by the various environmental changes for all of our ERC classifications (Fig. 10). Our analysis in Figs. 7 10 indicates that the internal dynamics and environmental conditions may both be important in the CEM cases, and that the NRC cases are strongly influenced by the large-scale external dynamics. The ERC cases experienced the least amount of variation in the largescale environment, which is suggestive that internal dynamics may be playing the pivotal role in governing inner-core intensity and structure changes.

\section{Summary}

An objective method is developed to categorize concentric eyewall (CE) structures in western North Pacific typhoons using the NRL SSM/I and TMI satellite imagery database. There are $77 \mathrm{CE}$ typhoons and $95 \mathrm{CE}$ cases identified from 26774 satellite images between 1997 and 2011. Excluding the cases that are close to landfall and cases without temporal resolution of $12 \mathrm{~h}$ or higher, $70 \mathrm{CE}$ cases were studied for the structural and intensity changes. The primary findings are as follows.

1) Three CE types are categorized: $C E$ with an eyewall replacement cycle (ERC; 37 cases, $53 \%$ ), CE with no replacement cycle (NRC; 17 cases, 24\%), and CE that is maintained for an extended period of time (CEM; 16 cases, 23\%). The inner (outer) eyewall dissipates in the ERC (NRC) type in less than $20 \mathrm{~h}$ after $\mathrm{CE}$ formation. The CEM type maintains its CE structure for more than $20 \mathrm{~h}$ (mean duration time is $31 \mathrm{~h}$ ).

2) The no-CE typhoons with intensity category 4 or above and far from land (NCE) have increased intensity and convection before they reach their maximum intensity and decreased intensity and convection afterward. The $\mathrm{CE}$ storms show quite different behavior than that of the NCE storms in terms of the structural and intensity change. This is illustrated with the $T$-Vmax diagram, in which the time sequence of intensities and the convective

TABLE 2. The average and standard deviation (SD) of zonal, meridional, and total translation speed in ERC, CEM, and NRC cases between $\mathrm{CE}$ formation and $24 \mathrm{~h}$ after $\mathrm{CE}$ formation.

\begin{tabular}{llccc}
\hline \hline & & ERC & CEM & NRC \\
\hline Zonal translation speed $\left(\mathrm{m} \mathrm{s}^{-1}\right)$ & Avg & 4.7 & 3.2 & 4.6 \\
& SD & 3.0 & 2.1 & 1.8 \\
Meridional translation & Avg & 3.4 & 2.9 & 4.8 \\
$\quad$ speed $\left(\mathrm{m} \mathrm{s}^{-1}\right)$ & SD & 2.5 & 1.3 & 2.2 \\
Avg translation speed $\left(\mathrm{m} \mathrm{s}^{-1}\right)$ & & 5.9 & 4.4 & 6.4 \\
SD $\left(\mathrm{m} \mathrm{s}^{-1}\right)$ & & 2.9 & 1.5 & 2.6 \\
\hline
\end{tabular}


(a)
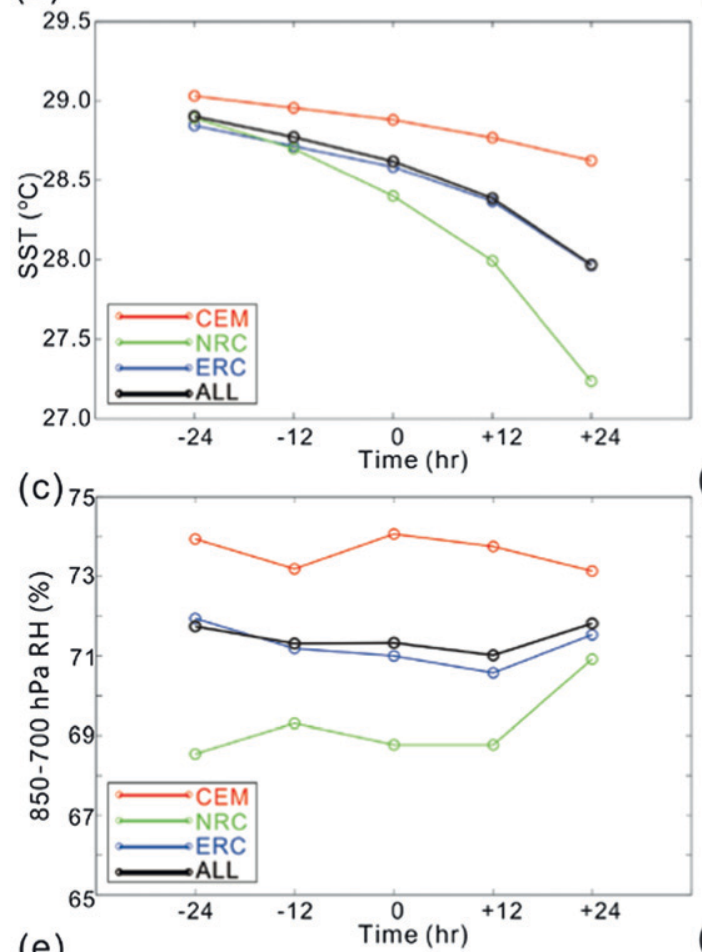

(e)

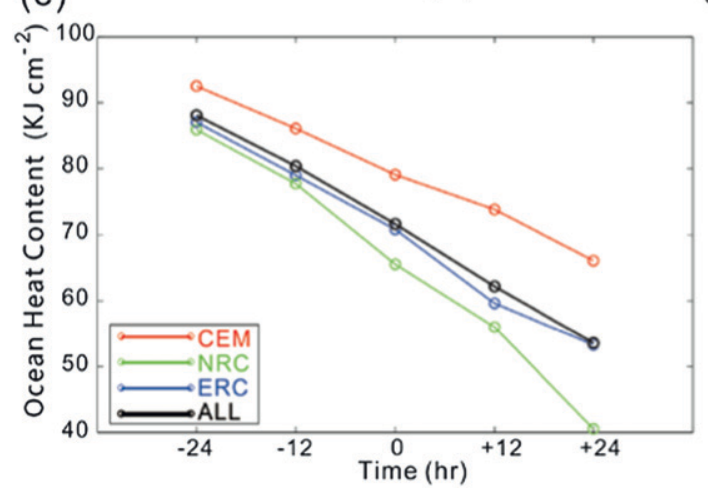

(b)

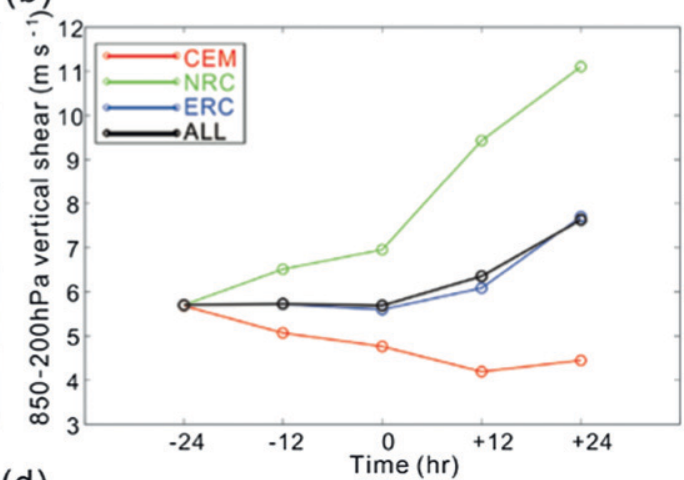

(d)

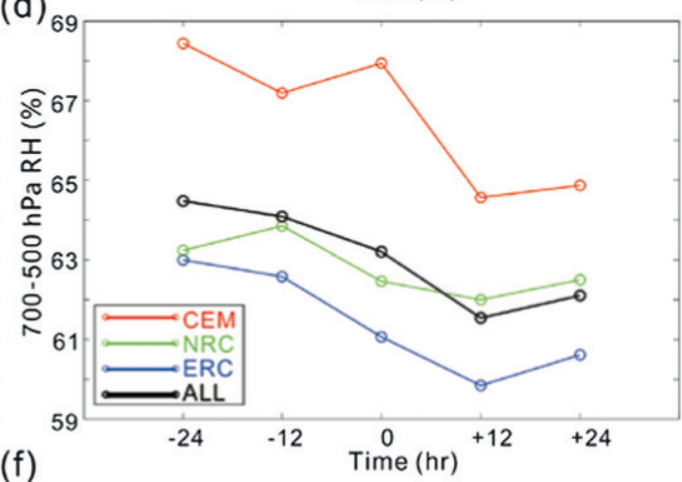

(f)

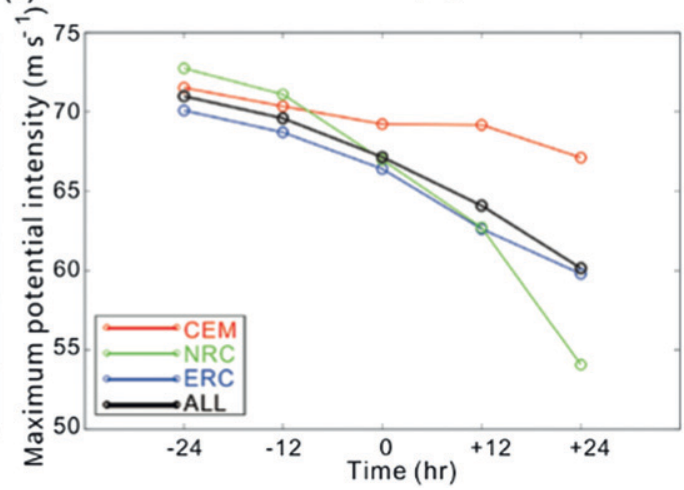

FIG. 10. The time series of (a) SST, (b) 850-200-hPa vertical shear, (c) 850-700-hPa RH, (d) 700-500-hPa RH, (e) ocean heat content, and (f) maximum potential intensity for ERC (blue), CEM (red), NRC (green), and all cases (black) $24 \mathrm{~h}$ before and after $\mathrm{CE}$ formation. The composite was done relative to the time of CE formation.

activity (CA) are shown. The CA is computed as the areal averaged $T_{B}$ contrast to the background centered at the eye. While the storm intensity weakens after CE formation, the CA is maintained or slightly increased in the ERC and CEM cases. This is in contrast to the NRC cases, where both CA and intensity decrease.

3) The CEM cases have relatively high-intensity, large widths of both the moat and outer eyewall that last for a long duration time. The moat size and outer eyewall width in the CEM cases are approximately $50 \%$ larger than that in the ERC and NRC cases. Our analysis suggests that vortex-scale filamentation dynamics lead to the organization of moat size. The CEM type may be simply end up taking much longer time to contract because of their larger scale. On the other hand, the large widths of the moat and outer eyewall may reduce the barotropic instabilities of $\mathrm{CE}$ storms thus maintain the CE structures for a long time. The large moat size in the CEM cases may have an impact on the convection and subsidence in both eyewalls. The interference between the convection/ subsidence couplet of the inner and outer eyewalls may be reduced when the moat size is very large. The 
large moat size may assist the inner core by suppressing potentially competing convection while the subsidence concentrated radial outward making it less likely to penetrate to the eyewall. Since the CEM cases are with long duration of significant storm size, the dynamics require more study in the future.

4) Most CEM cases in the western North Pacific are located to the west of $140^{\circ} \mathrm{E}$ with the smallest northward translation speed of $2.9 \mathrm{~m} \mathrm{~s}^{-1}$. The NRC cases often have fast northward translation speeds and are located in higher latitudes. The high vertical wind shear, low sea surface temperature (SST), and low relative humidity $(\mathrm{RH})$ act to weaken the convective activity for these cases. On the other hand, the CEM cases occur in environments of low vertical wind shear, high SST, and high RH. Our results suggest that both the internal dynamics and environmental conditions are important in the CEM cases, and the NRC cases are more influenced by the largescale external dynamics. The ERC cases, on the other hand, may be dominated by the internal dynamics because of the less appreciable change of the environment.

Acknowledgments. We thank Drs. Ching-Hwan Liu and Chih-Pei Chang for their original $\mathrm{T}_{\mathrm{B}}$-color switch code and suggestions. We thank Dr. M. DeMaria for providing the Statistical Typhoon Intensity Prediction Scheme (STIPS) data. The satellite microwave images were made available by the Naval Research Laboratory Marine Meteorology Division in Monterey, California. We also thank Wayne Schubert for helpful suggestion for CE determination and Robert G. Fovell for his advice and comments. The authors thank Jonathan Vigh and two anonymous reviewers for their very helpful comments. This research was supported by Taiwan's National Research Council through Grants NSC 101-2625M-002-010, NSC100-2111-M-002-004-MY3, NSC1002628-M-002-019, and NSC98-2745-M-002-008-MY3 to National Taiwan University; Grants 10R-70604-1 and 101R-70604-1 from the National Taiwan University; Grant 101R891404 from Taida Institute for Mathematical Sciences; and by the U.S. Office of Naval Research NICOP Grant N62909-11-1-7096.

\section{REFERENCES}

Barnes, G. M., and M. D. Powell, 1995: Evolution of the inflow boundary layer of Hurricane Gilbert (1988). Mon. Wea. Rev., 123, 2348-2368.

— E. E. J. Zipser, D. Jorgensen, and F. Marks Jr., 1983: Mesoscale and convective structure of a rainband. J. Atmos. Sci., 40, $2125-2137$.
Black, M. L., and H. E. Willoughby, 1992: The concentric eyewall cycle of Hurricane Gilbert. Mon. Wea. Rev., 120, 947-957.

DeMaria, M., and J. Kaplan, 1999: An updated Statistical Hurricane Intensity Prediction Scheme (SHIPS) for the Atlantic and eastern North Pacific basins. Wea. Forecasting, 14, 326-337.

_-, M. Mainelli, L. K. Shay, J. A. Knaff, and J. Kaplan, 2005: Further improvement to the Statistical Hurricane Intensity Prediction Scheme (SHIPS). Wea. Forecasting, 20, 531-543.

Dritschel, D. G., and D. W. Waugh, 1992: Quantification of the inelastic interaction of unequal vortices in two-dimensional vortex dynamics. Phys. Fluids, 4A, 1737-1744.

Emanuel, K., 1997: Some aspects of hurricane inner-core dynamics and energetics. J. Atmos. Sci., 54, 1014-1026.

_ 2000: A statistical analysis of tropical cyclone intensity. Mon. Wea. Rev., 128, 1139-1152.

Hawkins, J. D., and M. Helveston, 2004: Tropical cyclone multiple eyewall characteristics. Preprints, 26th Conf. on Hurricane and Tropical Meteorology, Miami, FL, Amer. Meteor. Soc., P1.7. [Available online at https://ams.confex.com/ams/26HURR/ techprogram/paper_76084.htm.]

- , and - 2008: Tropical cyclone multiple eyewall characteristics. Preprints, 28th Conf. on Hurricanes and Tropical Meteorology, Orlando, FL, Amer. Meteor. Soc., 14B.1. [Available online at https://ams.confex.com/ams/28Hurricanes/ techprogram/paper_138300.htm.]

— periment missions and post-mission analysis with satellite digital data and products. Bull. Amer. Meteor. Soc., 92, 10091022.

, T. F. Lee, F. J. Turk, C. Sampson, J. Kent, and K. Richardson, 2001: Real-time Internet distribution of satellite products for tropical cyclone reconnaissance. Bull. Amer. Meteor. Soc., 82, 567-578.

, M. Helveston, T. F. Lee, F. J. Turk, K. Richardson, C. Sampson, J. Kent, and R. Wade, 2006: Tropical cyclone multiple eyewall characteristics. Preprints, 27th Conf. on Hurricane and Tropical Meteorology, Monterey, CA, Amer. Meteor. Soc., 6B.1. [Available online at http://ams.confex. com/ams/27Hurricanes/techprogram/paper_108864.htm.]

Hence, D. A., and R. A. Houze Jr., 2012: Vertical structure of tropical cyclones with concentric eyewalls as seen by TRMM precipitation radar. J. Atmos. Sci., 69, 1021-1036.

Huang, Y.-H., M.-T. Montgomery, and C.-C. Wu, 2012: Concentric eyewall formation in Typhoon Sinlaku (2008). Part II: Axisymmetric dynamical processes. J. Atmos. Sci., 69, 662-674.

Kidder, S. Q., M. D. Goldbrig, R. M. Zehr, M. DeMaria, J. F. W. Purdom, C. S. Veldon, N. C. Grodg, and S. J. Kusselson, 2000: Satellite analysis of tropical cyclones using Advanced Microwave Sounding Unit (AMSU). Bull. Amer. Meteor. Soc., 81, 1241-1259.

Knaff, J. A., J. P. Kossin, and M. DeMaria, 2003: Annular hurricanes. Wea. Forecasting, 18, 204-223.

_ C. R. Sampson, and M. DeMaria, 2005: An operational statistical typhoon intensity prediction scheme for the western North Pacific. Wea. Forecasting, 20, 688-699.

Kossin, J. P., and M. Sitkowski, 2009: An objective model for identifying secondary eyewall formation in hurricanes. Mon. Wea. Rev., 137, 876-892.

— W. H. Schubert, and M. T. Montgomery, 2000: Unstable interaction between a hurricane's primary eyewall and a secondary ring of enhanced vorticity. J. Atmos. Sci., 57, 3893-3917. 
Kummerow, C., W. Barnes, T. Kozu, J. Shiue, and J. Simpson, 1998: The Tropical Rainfall Measuring Mission (TRMM) sensor package. J. Atmos. Oceanic Technol., 15, 809-817.

Kuo, H.-C., G. T.-J. Chen, and C.-H. Lin, 2000: Merger of tropical cyclones Zeb and Alex. Mon. Wea. Rev., 128, 2967-2975.

—, L.-Y. Lin, C.-P. Chang, and R. T. Williams, 2004: The formation of concentric vorticity structures in typhoons. J. Atmos. Sci., 61, 2722-2734.

— W. W. Schubert, C.-L. Tsai, and Y.-F. Kuo, 2008: Vortex interactions and barotropic aspects of concentric eyewall formation. Mon. Wea. Rev., 136, 5183-5198.

— C.-P. Chang, Y.-T. Yang, and H.-J. Jiang, 2009: Western North Pacific typhoons with concentric eyewalls. Mon. Wea. Rev., 137, 3758-3770.

$\longrightarrow$, — , and C.-H. Liu, 2012: Convection and rapid filamentation in Typhoon Sinlaku during TCS-08/T-PARC. Mon. Wea. Rev., 140, 2806-2817.

Maclay, K. S., M. DeMaria, and T. H. Vonder Haar, 2008: Tropical cyclone inner-core kinetic energy evolution. Mon. Wea. Rev., 136, 4882-4898.

McNoldy, B. D., 2004: Triple eyewall in Hurricane Juliette. Bull. Amer. Meteor. Soc., 85, 1663-1666.

Montgomery, M. T., and R. J. Kallenbach, 1997: A theory for vortex Rossby-waves and its application to spiral bands and intensity changes in hurricane. Quart. J. Roy. Meteor. Soc., 123, 435-465.

Musgrave, K. D., R. K. Taft, J. L. Vigh, B. D. McNoldy, and W. H. Schubert, 2012: Time evolution of the intensity and size of tropical cyclones. J. Adv. Model. Earth Syst., 4, M08001, doi:10.1029/2011MS000104.

Nong, S., and K. A. Emanuel, 2003: A numerical study of the genesis of concentric eyewalls in hurricane. Quart. J. Roy. Meteor. Soc., 129, 3323-3338.

Olander, T. L., and C. S. Velden, 2007: The advanced Dvorak technique: Continued development of an objective scheme to estimate tropical cyclone intensity using geostationary infrared satellite imagery. Wea. Forecasting, 22, 287-298.

Peng, J., T. Li, and M. S. Peng, 2009: Formation of tropical cyclone concentric eyewalls by wave-mean flow interactions. $A d v$. Geosci., 10, 57-71.

Poe, G., 1990: Optimum interpolation of imaging microwave radiometer data. IEEE Trans. Geosci. Remote Sens., 28, 800-810.

Qiu, X., Z.-M. Tan, and Q. Xiao, 2010: The roles of vortex Rossby waves in hurricane secondary eyewall formation. Mon. Wea. Rev., 138, 2092-2109.
Rozoff, C. M., W. H. Schubert, B. D. McNoldy, and J. P. Kossin, 2006: Rapid filamentation zones in intense tropical cyclones. J. Atmos. Sci., 63, 325-340.

- - $\longrightarrow$, and J. P. Kossin, 2008: Some dynamical aspects of hurricane eyewall replacement cycles. Quart. J. Roy. Meteor. Soc., 134, 583-593.

Samsury, C. E., and E. J. Zipser, 1995: Secondary wind maxima in hurricanes: Airflow and relationship to rainbands. Mon. Wea. Rev., 123, 3502-3517.

Schubert, W. H., C. M. Rozoff, J. L. Vigh, B. D. McNoldy, and J. P. Kossin, 2007: On the distribution of subsidence in the hurricane eye. Quart. J. Roy. Meteor. Soc., 133, 595-605.

Sitkowski, M., J. P. Kossin, and C. M. Rozoff, 2011: Intensity and structure changes during hurricane eyewall replacement cycles. Mon. Wea. Rev., 139, 3829-3847.

Spencer, R. W., H. M. Goodman, and R. E. Hood, 1989: Precipitation retrieval over land and ocean with the SSM/I: Identification and characteristics of the scattering signal. J. Atmos. Oceanic Technol., 6, 254-273.

Terwey, W. D., and M. T. Montgomery, 2008: Secondary eyewall formation in two idealized, full-physics modeled hurricanes. J. Geophys. Res., 113, D12112, doi:10.1029/2007JD008897.

Velden, C. S., and Coauthors, 2006: The Dvorak tropical cyclone intensity estimation technique: A satellite-based method that has endured for over 30 years. Bull. Amer. Meteor. Soc., 87, 1195-1210.

Vigh, J. L., J. A. Knaff, and W. H. Schubert, 2012: A climatology of hurricane eye formation. Mon. Wea. Rev., 140, 1405-1426.

Willoughby, H. E., 1979: Forced secondary circulations in hurricanes. J. Geophys. Res., 84 (C6), 3173-3183.

— namics of a disaster. Bull. Amer. Meteor. Soc., 77, 543-549.

_ J. A. Clos, and M. G. Shoreibah, 1982: Concentric eye walls, secondary wind maxima, and the evolution of the hurricane vortex. J. Atmos. Sci., 39, 395-411.

Wimmers, A. J., and C. S. Velden, 2010: Objectively determining the rotational center of tropical cyclones in passive microwave satellite imagery. J. Appl. Meteor. Climatol., 49, 2013-2034.

Zhou, X., and B. Wang, 2009: From concentric eyewall to annular hurricane: A numerical study with the cloud-resolving WRF model. Geophys. Res. Lett., 36, L03802, doi:10.1029/ 2008 GL036854.

, and - 2011: Mechanism of concentric eyewall replacement cycles and associated intensity change. J. Atmos. Sci., 68, 972-988. 